\title{
Gradhiva
}

GRADHIV

Revue d'anthropologie et d'histoire des arts

$22 \mid 2015$

Cosmos

\section{Petits récits destinés à joindre les deux bouts des particules au cosmos - en passant par la Suisse}

Short narratives to link extremes: from particles to the cosmos via Switzerland

\section{Sophie Houdart}

\section{(2) OpenEdition}

Journals

Édition électronique

URL : http://journals.openedition.org/gradhiva/3054

DOI : 10.4000/gradhiva.3054

ISSN : 1760-849X

Éditeur

Musée du quai Branly Jacques Chirac

Édition imprimée

Date de publication : 1 octobre 2015

Pagination : 106-135

ISBN : 978-2-35744-092-0

ISSN : 0764-8928

Référence électronique

Sophie Houdart, «Petits récits destinés à joindre les deux bouts des particules au cosmos - en

passant par la Suisse », Gradhiva [En ligne], 22 | 2015, mis en ligne le 01 octobre 2018, consulté le 23 avril 2019. URL : http://journals.openedition.org/gradhiva/3054 ; DOI : 10.4000/gradhiva.3054

(c) musée du quai Branly 


\section{$y=$}

With

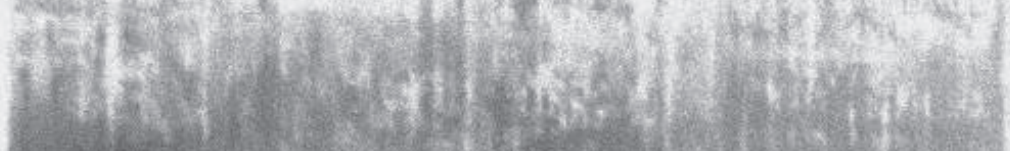
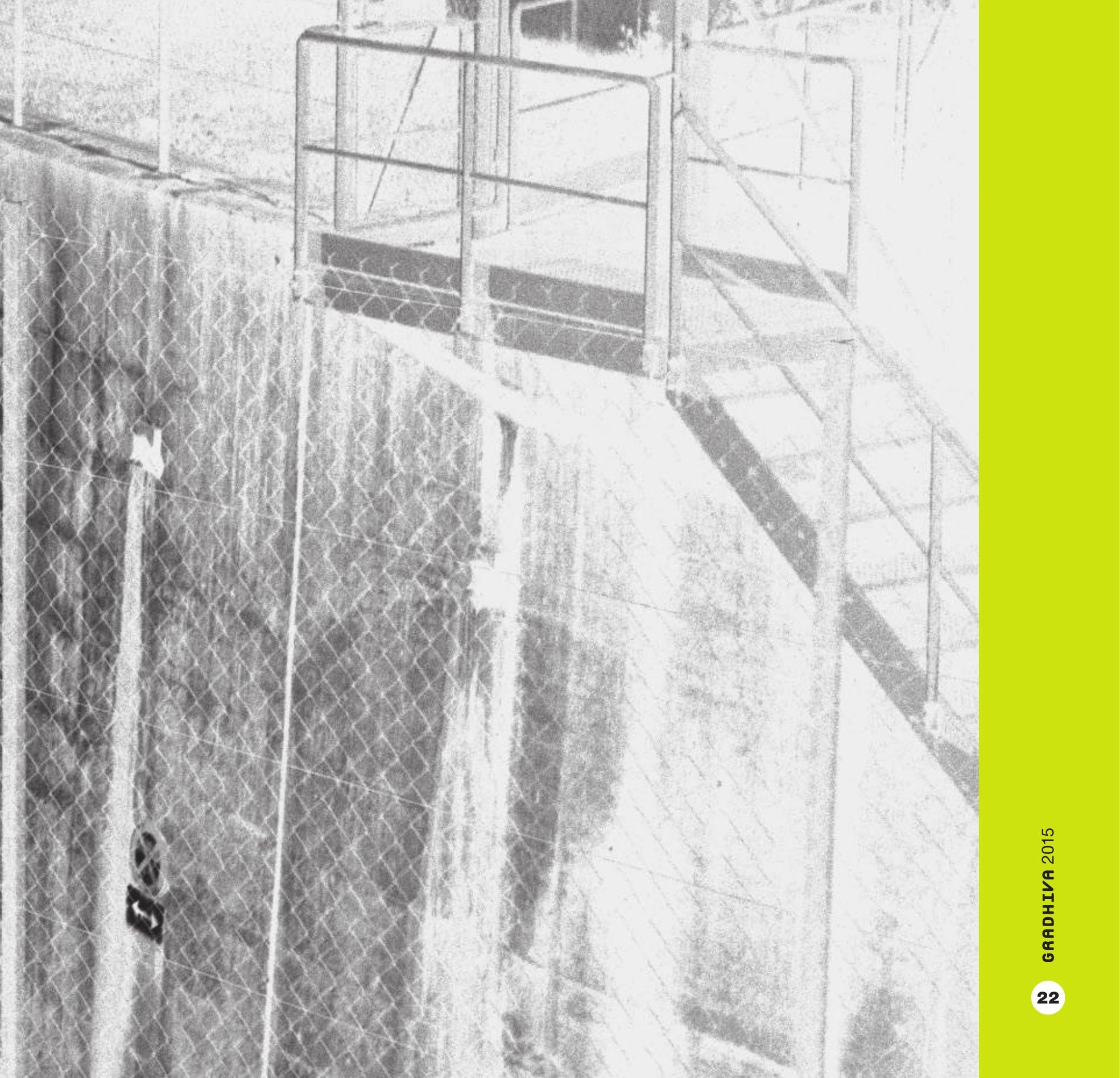

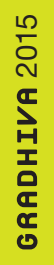

(x)

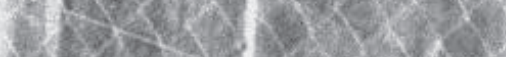

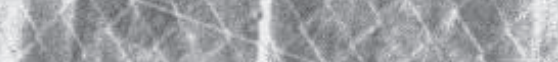

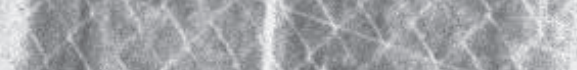

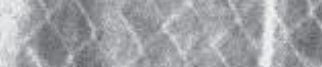




\section{Petits récits destinés à joindre les deux bouts des particules au cosmos - en passant par la Suisse}

par Sophie Houdart

D'où gagner le sentiment de soi dans l'univers? Partant du sentiment océanique cher au romancier Romain Rolland, cet article se propose de rendre compte, sous forme de petits récits, d'une enquête menée au CERN sur le Grand collisionneur de hadrons (LHC). Au regard de la littérature qui dresse en droite ligne une relation des particules au cosmos et présente la machine comme transcendance, il se situe à l'échelle humaine, au niveau de ce qu'il faut bel et bien pour connecter les unes à l'autre et éprouver le sentiment de ce qui nous déborde. 


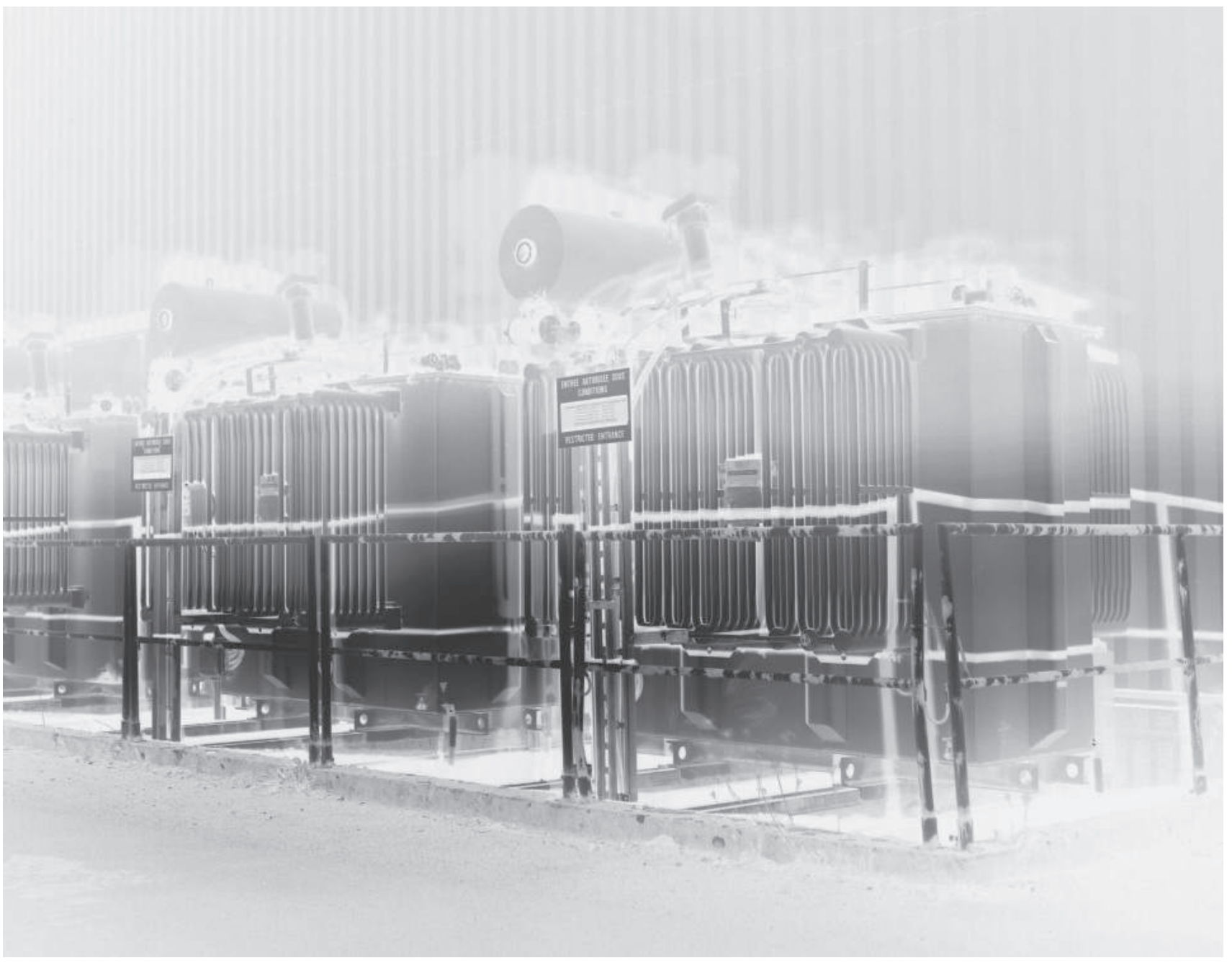

fig. 1

Propagation de la

Monotonie, CERN, 2011

(c) Grégoire Eloy / F93.
Romain Rolland à Sigmund Freud Villeneuve (Vaud) villa Olga, 5 décembre 1927

Cher ami respecté,

Je vous remercie d'avoir bien voulu m'envoyer votre lucide et vaillant petit livre. Avec un calme bon sens, et sur un ton modéré, il arrache le bandeau des éternels adolescents, nous tous, dont l'esprit amphibie flotte entre l'illusion d'hier et... l'illusion de demain.

Votre analyse des religions est juste. Mais j'aurais aimé à vous voir faire l'analyse du sentiment religieux spontané ou, plus exactement, de la sensation religieuse, qui est toute différente des religions proprement dites, et beaucoup plus durable.

J'entends par là: - tout à fait indépendamment de tout dogme, de tout Credo, de toutes organisations d'Église, de tout Livre Saint, de toute espérance en une survie personnelle, etc. -, le fait simple et direct de la sensation de l'«éternel » (qui peut très bien n'être pas éternel, mais simplement sans bornes perceptibles, et comme océanique). [...] Je suis moi-même familier avec cette sensation. Tout au long de ma vie, elle ne m'a jamais manqué; et j'y ai toujours trouvé une source 
de renouvellement vital. En ce sens, je puis dire que je suis profondément «religieux» - et sans que cet état constant (comme une nappe d'eau que je sens affleurer sous l'écorce) nuise en rien à mes facultés critiques et à ma liberté de les exercer - fût-ce contre l'immédiateté de cette expérience intérieure. Ainsi, je mène de front, sans gêne et sans heurt, une vie "religieuse" (au sens de cette sensation prolongée) et une vie de raison critique (qui est sans illusion)...

J'ajoute que ce sentiment "océanique" n'a rien à voir avec mes aspirations personnelles. Personnellement, j'aspire au repos éternel; la survie ne m'attire aucunement. Mais le sentiment que j'éprouve m'est imposé comme un fait. C'est un contact. - Et comme je l'ai reconnu, identique (avec des nuances multiples), chez quantité d'âmes vivantes, il m'a permis de comprendre que là était la véritable source souterraine de l'énergie religieuse; - qui est ensuite captée, canalisée et desséchée par les Églises: au point qu'on pourrait dire que c'est à l'intérieur des Églises (quelles qu'elles soient) qu'on trouve le moins de vrai sentiment «religieux».

Éternelle confusion des mots, dont le même, ici, tantôt signifie obéissance ou foi à un dogme, ou à une parole (ou à une tradition), tantôt: libre jaillissement vital.

Veuillez croire, cher ami, à mon affectueux respect

Romain Rolland (Vermorel et Vermorel 1993: 303-304)

C'est ainsi que Romain Rolland répondait à Sigmund Freud qui venait de lui faire parvenir L'Avenir d'une illusion. La conversation qu'entretinrent les deux hommes se prolongea, souvent par publications décalées, dans les années qui suivirent (Malaise dans la civilisation constituera la réponse restée la plus célèbre de ces échanges). Elle portait intrinsèquement sur les formalisations dogmatiques et sur les superstitions, dont Freud comme Rolland se voulaient les pourfendeurs - mais chacun à sa façon. "Situant les racines métapsychologiques de la religion dans le passé infantile de l'homme" (ibid.: 299), Freud voyait dans tout débordement de soi un point d'appui ou un remède à la détresse humaine, qu'il était nécessaire de surmonter pour entrer dans l'âge adulte. Au contraire, Rolland faisait valoir l'expérience vécue et difficile à stabiliser de ces moments, hétérogènes, au cours desquels pouvait s'éprouver le sentiment plein du monde et du cosmos: se perdre dans l'horizon de la mer ou bien dans l'étendue immense s'offrant au regard du montagnard, méditer... Emprunté à la mystique de I'Inde sur laquelle Rolland avait commencé à travailler (La Vie de Ramakrishna, publiée en 1943, entre autres), le concept de sentiment océanique servait à décrire les états, parfois éphémères, de communion avec une immensité, «la personnalité [s'y dissolvant] comme un grain de sel dans la mer; mais au même moment, l'infini de la mer [semblant] être contenu dans le grain de sable» (Koestler 1945: 270). Plus que de sensation, Rolland parle de "contact» pour désigner la rencontre entre deux réalités - deux échelles - a priori incommensurables et qui se croisent soudain, pour un temps donné. Cela peut ne pas arriver, ou cela peut arriver suivant des modalités très diverses. 
1. Cette enquête, commanditée par le Centre de culture scientifique, technique et industrielle de la Seine Saint-Denis, F93, a été réalisée de juin 2011 à janvier 2012. Intitulée "Propagation de la monotonie ", elle a été menée conjointement avec Grégoire Eloy, photographe (dont le travail illustre le présent article), et Stéphane Sautour, artiste plasticien. Voir http://www.f93.fr/ pour les résultats de cette collaboration. Je me permets également de renvoyer à Houdart 2015

2. Martin Beech fait référence à l'ouvrage de C.S. Lewis, publié en 1964 The Discarded Image, Cambridge University Press.
D'où, encore, gagner le sentiment de soi dans l'univers? Que me faut-il pour gagner la sensation ou la compréhension vive que quelque chose me déborde, une chose à laquelle pourtant je suis liée, connectée intimement? En m'appuyant sur l'enquête que j'ai menée récemment au Conseil européen pour la recherche nucléaire (CERN, aujourd'hui Organisation européenne pour la recherche nucléaire) sur le Large Hadron Collider (LHC), je voudrais montrer ici que le sentiment océanique ne requiert pas nécessairement - ou seulement - l'immédiateté de la contemplation transcendantale, mais peut naître d'un lent travail, parfois le long de chemins de traverse; comment, aussi, il est orchestré dans des dispositifs matériels, visuels ou rhétoriques chargés d'assurer le transport ou de susciter le point de contact délicat entre les particules, le cosmos et moi - vous tout autant ${ }^{1}$.

De fait, au CERN, des techniques d'enchantement prolifèrent, qui listent les innombrables atouts de la machine en usant de superlatifs destinés à rendre l'éloquence de l'aventure humaine en train de se jouer ici. Ainsi pourra-t-on lire sur le site internet de l'organisation, par exemple: «Le LHC, l'accélérateur de particules le plus grand et le plus puissant du monde, consiste en un anneau de $27 \mathrm{~km}$ de circonférence, lové $100 \mathrm{~m}$ sous terre. Formé d'aimants supraconducteurs et de structures accélératrices qui augmentent l'énergie des particules qui y circulent, il produit chaque jour, à l'intérieur de l'accélérateur, deux faisceaux de particules qui circulent en sens contraire à des énergies très élevées avant de rentrer en collision l'un avec l'autre. Les particules, lancées à 99,9999991 \% de la vitesse de la lumière, vont effectuer 11245 fois le tour de l'accélérateur par seconde et entrer en collision quelque 600 millions de fois par seconde. 》 Dans la littérature, les brochures de communication et les vidéos, l'exploit technique signifié par ces grands nombres est communément relayé par une ambition scientifique tout aussi magistrale: le LHC, en offrant la possibilité d'un «voyage dans la structure la plus profonde de la matière», a pour mission «la découverte des lois fondamentales qui régissent notre univers", et doit même permettre d'élucider «les premiers principes qui ont gouverné sa formation». "Tout est connecté», écrit l'astronome Martin Beech, et "dans le microcosme se réfléchit le macrocosme et vice-versa» (Beech 2010: VIII, notre traduction). À la manière des savants et astronomes du Moyen Âge ${ }^{2}$, les physiciens travailleraient aujourd'hui avec l'idée que le macrocosme, l'univers tel que nous le connaissons aujourd'hui, était déjà contenu dans le «microcosme primordial», dans la soupe de particules élémentaires, en même temps que ces particules évoluaient elles-mêmes déjà dans quelque chose qui ressemblait à un univers. "Le LHC, en brisant le nucleus en deux atomes principaux, nous permettra de remonter jusqu'à ces moments qui ont eu lieu juste $10^{-25}$ seconde après le Big Bang - un temps avant que la matière stable n'existe. 》 (Ibid.) Machine à remonter le temps, machine à tenir ensemble le microcosme et le macrocosme. Elle nous connecte au temps strict des origines - origines des conditions physiques qui ont permis l'agglomération du monde et l'apparition de la vie - et elle nous connecte au cosmos.

Pourtant, des particules accélérées à la vitesse de la lumière dans un tunnel cent mètres sous terre à la résolution des mystères du cosmos, le saut reste, concédons-le, difficile à faire pour qui n'a pas du modèle standard et de la déclinaison des quatre forces une vue parfaitement claire... II s'avère 
également périlleux à ceux qui, comme l'auteure de cet article, n'ont pas frémi en découvrant, enfants, la saga des étoiles, Star Wars, ni ne se sont sentis portés au-delà d'eux-mêmes, incapables d'étendre à ces confins leur territoire de jeu et d'exploration. Pour ceux-là, des dispositifs rhétoriques existent pour les y aider, au nombre desquels la technique récurrente qui consiste à mettre en équivalence des entités que rien ne semble devoir rapprocher. Dans A Zeptospace Odyssey. A Journey into the Physics of the LHC (Giudice 2010), Gian Francesco Giudice, physicien théorique au CERN, produit, de ce point de vue, une série d'équivalences particulièrement éloquente pour donner à penser les mesures du LHC à l'aune du très petit (à l'échelle du zepto, 10-21) comme à celle du très grand: on apprend ainsi que le LHC est «plus grand que le temple de Salomon" (ibid.: 120), qu'un dipôle est de même prix au kilo que le chocolat suisse (ibid.: 104), que l'énergie qui y est stockée équivaut à 2,4 tonnes de TNT ou 460 kilos de chocolat (ibid.: 116), qu'une caverne procure la même charge émotionnelle que les pyramides égyptiennes ou les cathédrales (ibid.: 131), qu'une pièce du détecteur CMS pèse 1920 tonnes mais aussi 400 éléphants d'Afrique (ibid.: 132)... Parmi toutes ces équivalences, l'on sent bien que certaines sont plus efficaces que d'autres. Traduire un dipôle en chocolat suisse n'informe pas tellement, si l'on y pense, ni sur le dipôle ni sur le chocolat. En revanche, traduire une caverne expérimentale en cathédrale est susceptible de produire un effet - c'est probablement la raison pour laquelle l'association LHC/ cathédrale est récurrente, et circule même parfaitement bien entre les physiciens, les ingénieurs, les chefs de chantier, les journalistes et les sociologues tentant de qualifier le LHC. L'analogie circule également parmi les anthropologues. Dans l'étude qu'elle réalise au milieu des années 1980 au sein de plusieurs laboratoires de physique des particules, l'anthropologue américaine Sharon Traweek explique l'extraordinaire implication de la physique et des physiciens dans nos sociétés actuelles par au moins deux raisons, très différentes: l'organisation, d'abord (la faculté des physiciens à s'organiser en communautés d'intérêt larges, aisément représentables devant les instances de décision internationales); «le pouvoir émotionnel de la cosmologie", ensuite, qui confère aux physiciens une aura digne de héros prométhéens en quête de la vérité sur les mystères de l'univers; les physiciens «donnent des nouvelles d'un autre monde: caché mais stable, cohérent et incorruptible. [...] L'échelle et le coût extraordinaires de la plupart des équipements de physique viennent renforcer la valeur culturelle [de ce gospel]. Les grands accélérateurs, par exemple, sont comme des cathédrales médiévales: libres des contraintes d'une analyse en termes de coûts et bénéfices." (Traweek 1988: 2, notre traduction) Comme ses consœurs médiévales, le LHC, cette cathédrale des temps modernes, offrirait un moyen de se connecter à l'immensité et aux mystères de l'univers, d'accéder à quelque chose qui nous dépasse largement et qui pourtant nous contient - l'expression moderne d'une transcendance.

La rhétorique, on le sent bien, oscille entre la célébration de l'artefact qui doit tout au génie humain (c'est une conquête sur les limites qui sont les nôtres) et la reconnaissance que cette chose-là nous dépasse tout autant que ce dont elle traite. Gigantesque par nécessité technique (il faut cela pour accélérer les particules à une vitesse proche de celle de la lumière), le LHC exprime les propriétés de l'objet qu'il contribue à élucider: les propriétés 


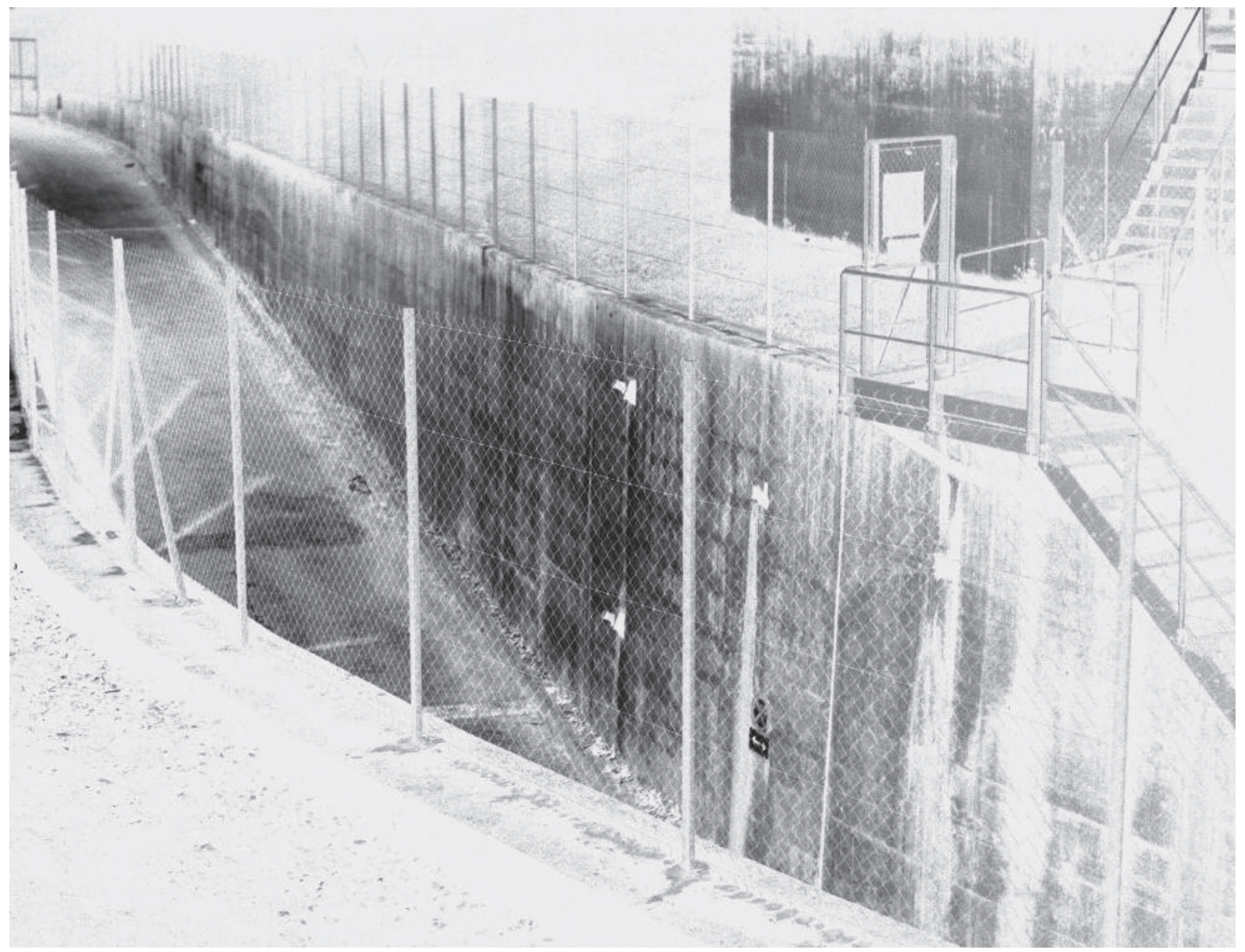

fig. 2

Propagation de la

Monotonie, CERN, 2011

(c) Grégoire Eloy / F93. 
du cosmos même, où se retrouvent indéfectiblement liées sa complexité, son instabilité et sa charge mystérieuse. D'une certaine façon, il est à la fois le temple et comme un dieu. C'est éloquent, soit, et le motif est connu. Le supplément d'âme, cependant, tient trop manifestement de l'artifice (Latour 2014) et la manœuvre seule ne peut suffire à m'emporter. Et elle ne peut suffire à me faire comprendre, non plus, comment est obtenu cet effet formidable que je ne peux manquer de noter dans le regard et les propos de mes interlocuteurs, physiciens, géomètres, ingénieurs, opérateurs, métrologues, qui prennent soin jour après jour du LHC : respect, fascination, adoration même.

Que faut-il donc pour qu'une image du cosmos tienne? À quelle échelle peut-on se placer pour éprouver (au double sens de sentir et de mettre à l'épreuve) que ce qui se joue au LHC compte pour nous? Pour répondre à ces questions, j'ai eu recours à une écologie des savoirs, qui a trouvé dans de petits récits sa forme la plus appropriée ${ }^{3}$. Ceux-ci, extraits de mes rencontres avec des géomètres et des opérateurs, sont destinés à faire voir le travail qu'ils accomplissent jour après jour pour obtenir et stabiliser le contact entre des choses situées à des échelles incommensurables.

\section{La machine et le sanglier}

Octobre 2011. Je retrouve Sacha et Bruno, géomètres au service site et patrimoine du CERN, à Ornex, sur la route entre Ferney-Voltaire et Gex, aux abords d'un site de casse automobile. Sont également présents trois techniciens du service électricité et du service connectique. Les deux propriétaires de la casse auto expliquent, plans en main, les projets qu'ils prévoient de réaliser. Terre à terre - on ne peut a priori imaginer place moins adéquate pour obtenir, de là, un regard sur quelque chose qui nous déborderait. En accord avec les normes environnementales, les propriétaires sont tenus de faire les aménagements nécessaires pour récupérer les eaux du parking et construire une dalle en béton là où il n'y a maintenant que de la terre. Ils ont donc déposé une déclaration d'intention de commencement de travaux (DICT) et ont été redirigés vers le CERN à cause de la servitude sur leur terrain. En effet, tout au long de la casse auto et dans la forêt qui la borde, le CERN a des réseaux qui regroupent, dans la même tranchée, fibres optiques et câbles à haute tension. Bruno m'explique que, dans la tranchée, passent la ligne de fibre optique qui traverse toute la zone et du courant de 66000 volts qui alimente différents points expérimentaux du CERN. «On va être encore plus stricts, raconte-t-il, sur les croisements de réseaux et les chantiers après l'épisode qu'il y a eu il y a un mois, ça a fait beaucoup de bruit. Les gars sont venus, ils avaient un poteau à remonter apparemment. C'était du côté de Crozet, du terrain de moto-cross. Le type du chantier est arrivé et a juste donné un grand coup de pioche! On a eu $10 \mathrm{~km}$ de fibres à changer... " Pendant que nous parlons, Sacha, dans les plans, essaie de repérer le tracé de la tranchée au-delà de la casse auto: elle part dans la forêt et son tracé devient invisible sur le plan en orthophoto.

M. LE PROPRIÉTAIRE: On voulait vous demander aussi si on pouvait prolonger ce merlon de terre qui court le long de la clôture. Si on pouvait le prolonger et le remonter de $80 \mathrm{~cm}$, contre les effractions. C'est qu'il y a de l'argent ici... et c'est aussi pour la sécurité des gens qui voudraient s'aventurer dans la casse auto.
3. Je me réfère sur ce point aux travaux de Tim Ingold (notamment Ingold 2000 et 2011) ainsi qu'à la réflexion ouverte en la matière par Isabelle Stengers: «... le champ de l'écologie des pratiques se définit d'abord et avant tout par le fait que la manière dont ces pratiques se présentent, se justifient, définissent leurs exigences et leurs obligations, et la manière dont elles sont décrites, dont elles sont susceptibles d'intéresser, dont elles doivent rendre compte à d'autres, sont interdépendantes et appartiennent à la même temporalité. Dès lors, tout argument, critique ou dénonciateur, justificatif ou incitatif, est un mode d'intervention qui vient s'ajouter à l'ensemble enchevêtré des manières dont les différents protagonistes s'adressent d'ores et déjà les uns aux autres. Mais la possibilité d'une écologie des pratiques exige de plus la viabilité d'un registre d'intervention qui affirme explicitement l'événement d'entre-capture que traduit et fait advenir tout point de vue reconnu comme pertinent. Elle exige donc ce que ce texte exige de ses lecteurs: l'abandon de l'opposition entre "description fidèle" et "fiction", comme aussi entre "constat" et "valeur", pour une démarche ouvertement constructiviste, c'est-à-dire qui résiste activement au plausible et au probable visés par les démarches qui se veulent neutres. ” (Stengers 1996: 97) 


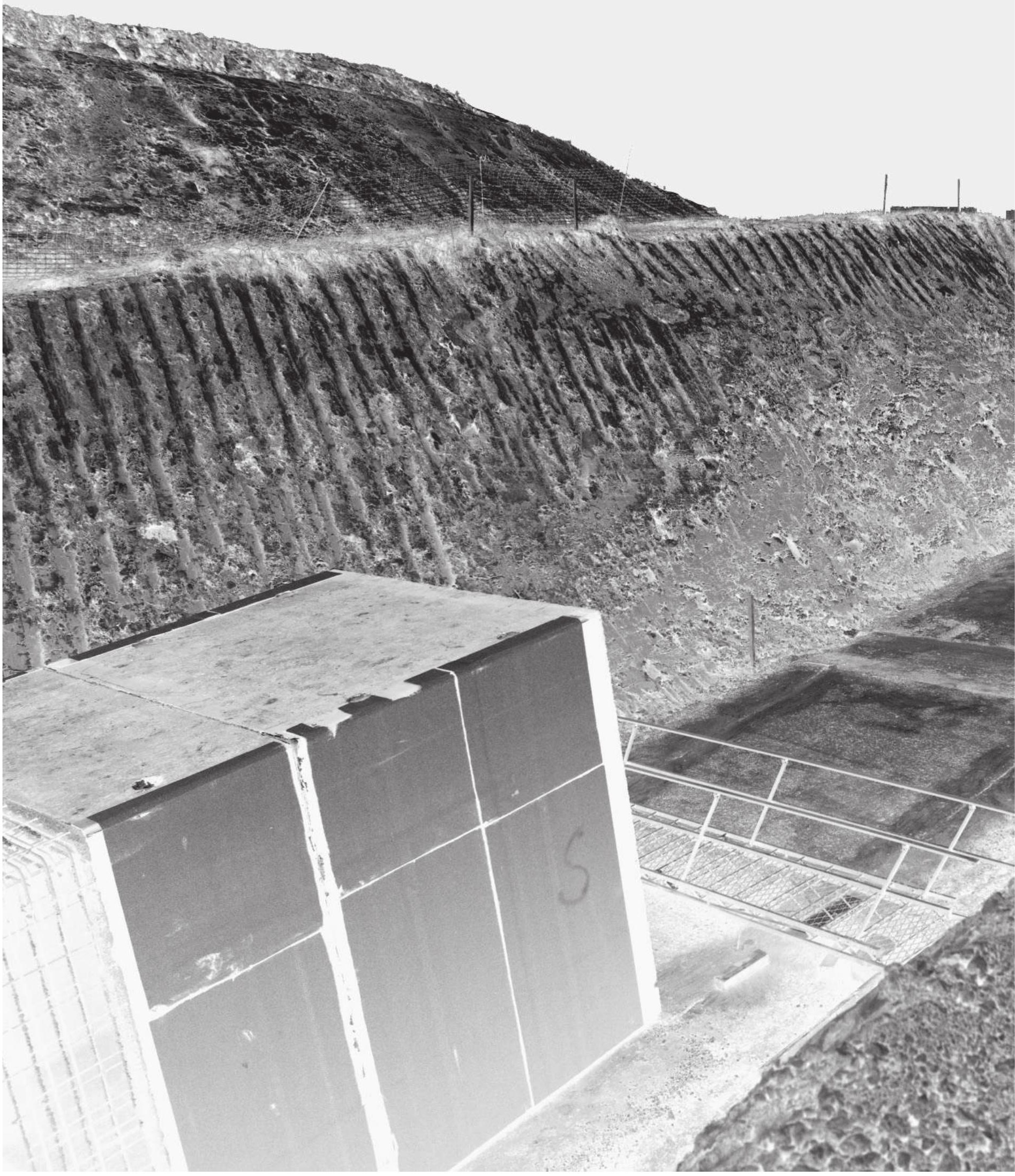




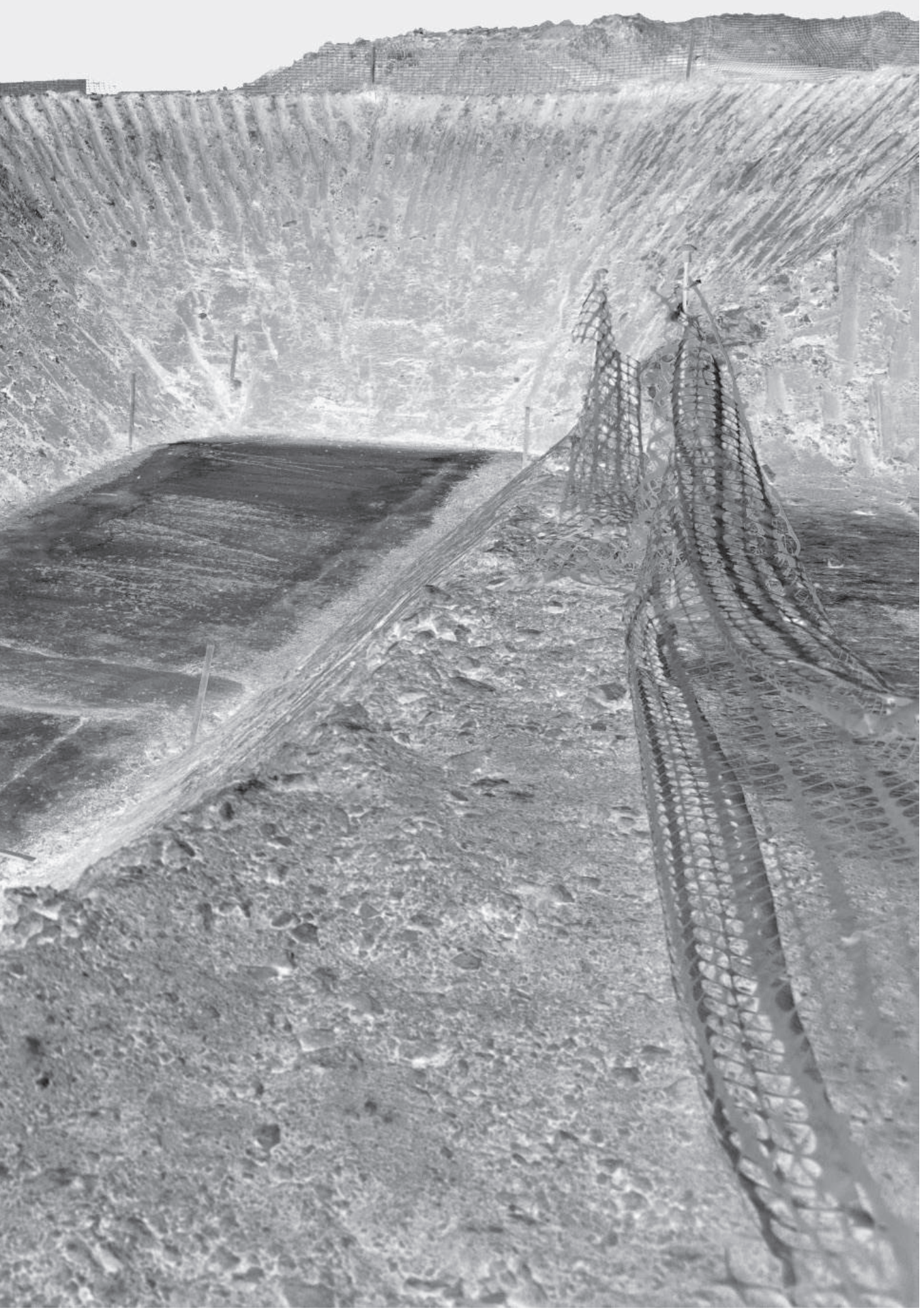


MME LA PROPRIÉTAIRE: Il faut que vous puissiez rentrer, là? Si vous devez revenir en urgence... II vous faudrait une barrière?

M. LE PROPRIÉTAIRE: Qu'est-ce qui pourrait faire que vous ayez à revenir en urgence? Vous ne prévoyez pas de rénovation de réseau? Parce que si vous nous dites que, dans deux ans, vous devez repasser...

PREMIER TECHNICIEN : Les câbles qui passent ici ne sont pas si vieux que ça... Il faut compter cinquante ans avant d'imaginer intervenir pour les rénover.

SECOND TECHNICIEN : Et maintenant on fait passer les câbles dans des fourreaux, on n'embête plus personne!

PREMIER TECHNICIEN: Mais c'est sûr qu'on ne peut pas dire aujourd'hui qu'on ne reviendra pas... Pour les fibres, il n'y a jamais d'urgence à venir les réparer à 3 heures du matin...

M. LE PROPRIÉTAIRE: Dès que vous nous donnez les documents, on commence les travaux. On a une pression énorme côté DRIR...

SACHA: II faudra faire attention, sur les plans on a une marge de $34 \mathrm{~cm}$ d'écart avec le nivellement général de la France (NGF). Et on n'est pas non plus en coordonnées Lambert...

\section{LE PROPRIÉTAIRE : On voudrait du Lambert et du NGF.}

SACHA: On pourra vous éditer les plans en Lambert, mais pour NGF... on vous donnera les conversions! Les géomètres du coin connaissent un peu la problématique CERN, de toute façon...

MME LA PROPRIÉTAIRE : À cet endroit, la tranchée part dans la forêt, il faudrait vérifier aussi. Ça bouge en forêt... Depuis qu'ils ont fait le chemin communal, il y a du passage. Plus loin dans la forêt il y a une gouille. Et avec les vélos, tout ça, il y a un endroit qui est maintenant une vraie mare pour les sangliers. Il faudrait voir si le terrain n'a pas bougé, qu'il n'y a pas d'affaissement...

Des sangliers, des sentiers forestiers, des propriétaires de casse auto... C'est bien toujours du LHC qu'il est question. Et c'est bien le cosmos qui s'étend encore à l'horizon. Mais cet horizon, ancré qu'il est sur un territoire singulier - rendu tel par la présence de la machine -, est soudain plus mondain et s'inscrit plus spontanément dans la dimension humaine. En forêt, «ça bouge», d'une vie peu concernée par le LHC qui, lui, doit pourtant la prendre en compte puisqu'il en dépend.

\section{Le vaisseau spatial et ses navigateurs}

Novembre 2011. La scène a lieu dans ce qui ressemble fort à une tour de contrôle, n'était que la vue panoramique sur laquelle elle ouvre est à l'intérieur et non à l'extérieur. Nous sommes dans le centre de contrôle central des expériences. C'est là qu'est paramétrée la machine, de là que sont 
orchestrés les faisceaux de particules. Les opérateurs «aux commandes» comparent volontiers leur travail à la conduite d'un vaisseau ou d'une voiture de course. La machine requiert de fonctionner en continu, vingt-quatre heures sur vingt-quatre, et des shifts de huit heures sont nécessaires pour la «surveiller». Le travail est décrit par certains physiciens, notamment ceux qui font de la théorie, comme «pas très glamour, il faut juste tourner un bouton! ». Mais comme nous allons le voir, la conduite de ce «vaisseau» singulier en appelle à bien d'autres compétences ${ }^{4}$.

II est 23 heures, je m'apprête à prendre le shift de nuit avec Louise et Jean-Paul. Aux quatre coins de l'immense salle, des îlots d'un quart de cercle formés par une lignée d'ordinateurs munis d'un ou deux écrans. Sur les murs au-dessus de ces îlots, de grands écrans affichent des mesures. On a un îlot pour les injecteurs, un pour l'un des premiers collisionneurs qui sert dorénavant de rampe de lancement pour les particules (le SPS), un autre qui gère l'eau, l'électricité et la cryogénie, et un dernier, le LHC. Dans chacun, deux personnes assurent leur tour de garde. C'est l'heure des passations, l'équipe précédente débriefe celle qui s'apprête à prendre son quart de nuit. Ils s'assoient tous les quatre en cercle, Louise et Jean-Paul écoutant attentivement les informations que leur transmettent leurs collègues.

De ce premier échange, je ne comprends rien, à peine la langue qui est parlée, un mélange très fondu de français et d'anglais. II est question de random, de cleaning. "C'était sur Beam 1?» Ils ont essayé de trouver la cause pendant une heure, ils ont fait un roll back. "C'est visiblement la cavité qui a des problèmes. " J'entends que ce problème, qui concerne l'un des deux faisceaux, Beam 1, et dont on n'identifie pas complètement la cause, empêche de "partir en physique». Les coordinateurs n'étaient pas d'accord, certains n'ont pas voulu risquer de compromettre un test de physique. J'entends beaucoup «remplir». J'entends deep de life time, qui sonne aussi comme un problème. Ce sont les «mêmes symptômes" qu'il y a quinze jours, et cela concerne la même cavité, fait remarquer Louise. Pour compléter le tableau des heures précédentes, la jeune femme dit que «plus ou moins en même temps, on a perdu des BLM parce qu'on a perdu la REV. Donc on a décidé d'avorter et de faire de la physique. On a aussi des problèmes pour injecter. Dans l'avant-dernière injection de faisceau, on a eu des oscillations. » J'entends aussi : «On a séparé les beams... manuellement, doucement, petit à petit... »

Bon. Se connecter au cosmos est une affaire visiblement technique, jamais totalement acquise et difficile à stabiliser.

La nuit commence tranquillement et s'annonce plus calme que la journée, selon mes interlocuteurs. Les particules ont été injectées et la machine «ronronne» doucement. Un peu plus tard, les quatre équipes se retrouvent autour de la grande table centrale où les membres de l'équipe LHCb ont organisé une petite fête après avoir atteint un de leurs objectifs. On sert des jus d'orange et on se partage un tiramisu. L'ambiance est intimiste. Je me présente: «Sophie, anthropologue, je suis venue enquêter sur le LHC, son rapport au sol, les échelles qu'il doit coordonner pour produire du cosmos une image claire. " Chacun, en retour, y va de ses quelques mots
4. Sur les compétences hétérogènes et distribuées requises pour conduire un vaisseau, voir Helmreich 2009 ou Hutchins 1995. 

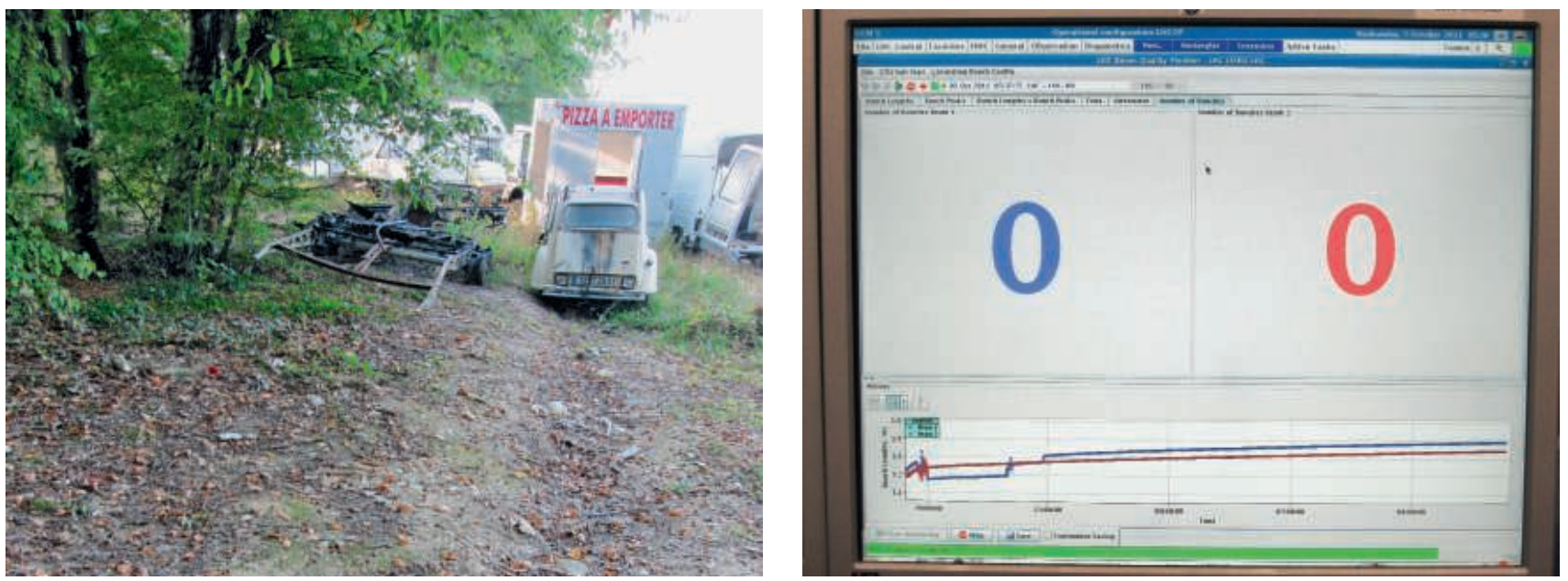

sur son îlot. Les faisceaux ont atteint leur vitesse de croisière et il semble qu'on ait le temps de discuter. De retour à notre poste dans l'îlot LHC, Louise m'explique qu'un faisceau de protons compte 1380 paquets. On compte aussi en trains, il y a des trains de 144 spaquets, d'autres de 72 paquets. Chaque train est susceptible de produire des effets dynamiques différents, «ils vont avoir une granularité différente». "Mon objectif, dit Louise, c'est de garder des caractéristiques stables sur l'ensemble du faisceau. Maintenant que la machine a de la maturité, on a du feed-back. On travaille avec ce feedback pour essayer de garder les paramètres les plus constants possibles. Mais à chaque fois on se pose la question: "Est-ce qu'aujourd'hui est un bon jour? Pas un bon jour?" C'est comme tous les pilotes, on a toujours le même circuit, on a toujours les mêmes voitures, mais il y en a un qui gagne "... Produire un bon faisceau et le rendre stable: c'est une question technique, qui va également dépendre de très nombreux paramètres, pas toujours contrôlables. Tout se passe comme si les faisceaux avaient une vie en propre, et que chacune de ces vies était irréductible aux autres. «Tout cela, me dit Louise, va dépendre de l'historique, de ce qui a été fait comme cycle magnétique avant par exemple. Et puis ce n'est pas pareil d'injecter au bout de deux heures ou au bout de cinq heures. On regarde ce qui s'est passé dans les vingt-quatre heures: "Il s'est passé ça, je peux m'attendre à avoir ça comme problème." ”

Dans le lot de ce qui peut faire la différence, les physiciens de faisceau comptent volontiers le fait d'avoir participé ou non à la période de démarrage de l'installation. «Ceux qui ont vécu ça vont avoir tendance à remplir step by step pour aller en collision. Alors que ceux qui arrivent juste vont être moins sensibles à ça, ils n'ont pas vécu toute la période de tests, les instabilités», explique Louise. Peut également jouer le fait de «venir de l'expérience», être physicien ou ingénieur - «Moi, dit Louise, je suis côté machine. » Après, il y a aussi les «impondérables». "Comme vendredi il y a quinze jours, un câble électrique a brûlé et c'est l'injecteur qui a sauté. Et il y a un mois, au cours de travaux réalisés sur le réseau, un technicien a donné un coup de pioche sur un câble. Le monsieur s'en est bien sorti, il a eu beaucoup de chance, le coup a porté entre la terre et le câble. Mais là, la machine, elle n'a pas aimé... » 

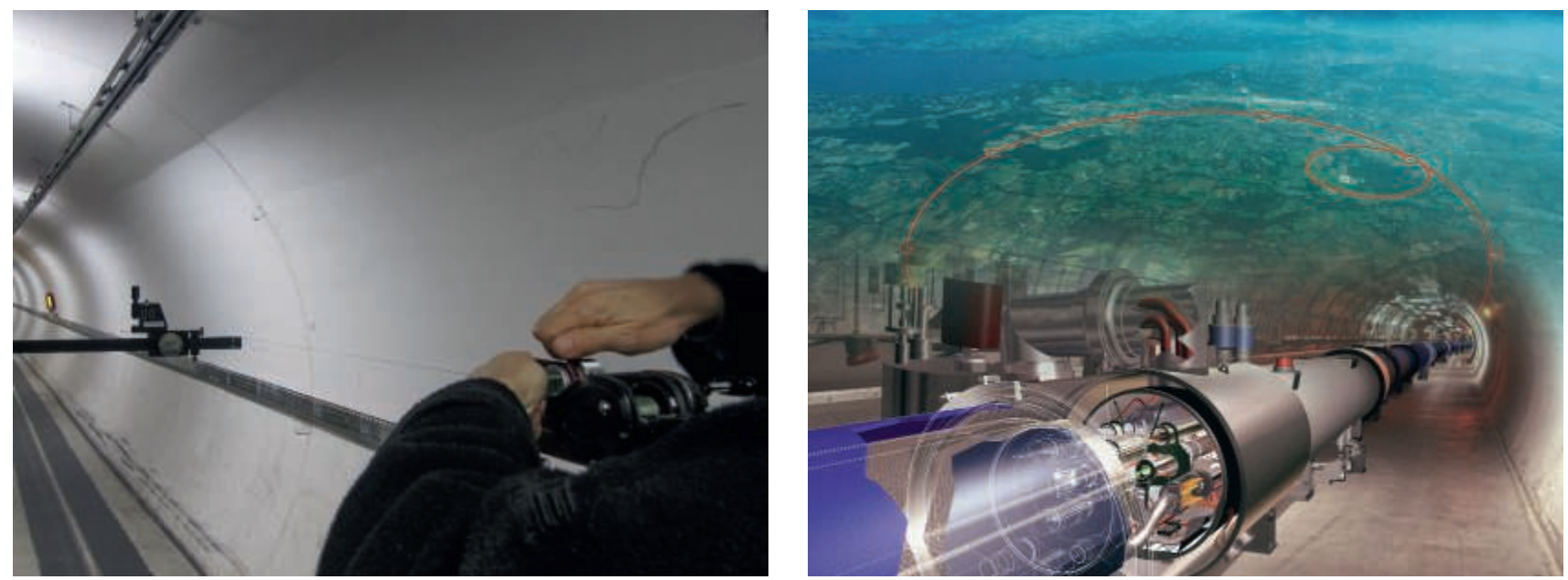

Et tandis que le temps s'écoule lentement, tandis que rien ne se passe de tous ces événements susceptibles d'advenir à tout moment, Louise et Jean-Paul continuent d'évoquer ces nuits qui «ne sont pas les mêmes", ces nuits agitées, chaotiques, "des nuits où on doit faire trois injections » par exemple. «On peut avoir des pics de vide», des phénomènes complètement aléatoires auxquels on ne peut rien, on perd le faisceau sans comprendre. On essaie des choses, on réduit l'intensité, mais «des fois, ça ne passe pas». Jean-Paul et Louise notent, comme pour eux-mêmes, que «c'est beaucoup par séries »: «Quand un équipement commence à avoir une défaillance, on va en avoir par séries. On a eu une série d'alimentations qui claquaient, maintenant on a une série avec des problèmes de vie du faisceau. On a eu aussi une série avec les kickers, les aimants rapides qui servent à l'aiguillage, et qui ne kickaient plus - et là, bizarrement, on n'en entend plus parler. On a eu aussi la série des UFO, pour Unidentified Falling Objects ou Unidentified Fast Objects, c'est selon, on dit l'un ou l'autre. On avait comme des petites bulles qui tombaient sur les faisceaux, induisant des pertes très rapides et les faisceaux disparaissaient. Et personne n'a jamais compris ce que c'était. II y a eu plein de tentatives d'explication et de manips pour tenter de les reproduire. Ça pouvait être de la poussière, on n'a jamais vraiment trop su. Quand on pousse la machine, diagnostique Louise, ça met en limite un certain nombre d'équipements. »

Tandis que, comme cette nuit, rien encore ne semble vouloir bouleverser l'homogénéité et déstabiliser la course des faisceaux, Louise me confie: «Mon truc à moi, ma psychose, c'est d'avoir une homogénéité dans les paquets. Ce n'est pas très scientifique, mais je préfère prendre le temps à l'injection pour avoir des paquets homogènes. C'est d'expérience. » Et lorsque, comme cette nuit, il n'y a pas grand-chose à faire, Louise "aime bien observer ", observer letemps d'optimisation entre deux opérations, faire des corrélations, regarder régulièrement les niveaux de perte. "Ça aide à comprendre s'il y a un dump après, une perte du faisceau. C'est ma façon d'apprendre, observer le faisceau. C'est comme vous, l'anthropologue. Sauf que moi, l'idée c'est d'observer les autres pour essayer d'homogénéiser. Je veux voir comment le faisceau réagit à telle situation. Qu'est-ce qui fait qu'on a, aujourd'hui, un faisceau qui perd plus que l'autre. Voir quelles actions on peut faire pour 
améliorer le faisceau. Ce qu'on peut tenter. Mais quoi qu'il en soit, c'est toujours le faisceau qui a le dernier mot. Le papier peut dire que le faisceau doit faire ça ou ça, si la machine ne veut pas... c'est mon côté technique. »

La nuit s'étire, il est 5 h 40 . Nous sommes plusieurs à sortir de la salle - pour la première fois de la nuit, en ce qui me concerne...-pour ranger des plats dans la cuisine et nettoyer la table. Lorsque je reviens, «tout a sauté, on a perdu la com!». Sur les écrans, Beam 1 et Beam 2 annoncent «zéro» là où, quelques minutes plus tôt, ils affichaient imperturbablement 1380 . Sur les différents écrans, des mesures se mettent au rouge un peu partout... C'est l'affolement! À l'îlot LHC, on cherche tout de suite quelle peut être la cause de ce down. Louise annonce tout de suite: «La cryo en a pris un coup, on n'aura pas le temps de reremplir... » - à un moment où tout le monde se félicitait, coupe de champagne à la main, du confort d'avoir un beam «stable comme ça»! L'«événement initial » est diagnostiqué au point RBA78. II s'agit d'un aimant dipôle. «Là, c'est du hardware, c'est une casse mécanique. L'expert est en train de regarder ce qui s'est passé. " Sur un écran qui diffuse en instantané des messages aux membres des quatre grandes expériences et sur l'ensemble du site, Louise écrit: “No beam before 8 h 30. " L'équipe suivante commence à arriver. Poursuivant un jeu de corrélations commencé plus tôt dans la nuit: «Bon... qui a ouvert sa porte de garage? » Louise passe à côté, dans l'îlot cryo: “Ouh là... c'est bien rouge... » Le téléphone sonne. Des différents centres de contrôle des expériences, les physiciens appellent pour savoir ce qui se passe et quand ils pourront «retourner en physique »... «Alors que là, ce n'est pas notre premier souci», commente Louise en aparté. Elle leur explique: «We recover the cryo first, it's gonna take a couple of hours », une heure pour la cryo donc, et une heure de pré-cycle, c'est ça, on n'aura pas de faisceau avant 8 heures au moins. Après avoir raccroché: «Si l'événement initial est plus grave qu'on ne pensait, il faudra faire un accès. " Plus tard, Louise au téléphone: «Bonjour, j’aurais besoin de faire un accès dans la machine. » Elle obtient les coordonnées de Régis, la personne d'astreinte qui doit être là dans l'heure, à qui il est demandé un accès au point 8. «C'est pour aller faire un reset sur le module TPS... Ah oui, il faut arriver avant les horaires officiels, là le LHC est en panne! Oui il faut aller chercher votre vélo, c'est en cellule 32, en plein milieu de l'arc. Vous y êtes dans quarante minutes, O.K., à 7 h 20. » Et de commenter après avoir raccroché: "Pas très motivé, le piquet... II faudra rappeler pour vérifier qu'il est bien parti. »

J'avoue être réellement captivée par le portrait du LHC tout en aspérités qui se dessine au centre de contrôle. Moi qui cherchais du relief dans l'univers plat qui dessine communément en ligne droite la relation du cosmos et des particules, me voilà servie. Les différentes opérations articulent des paramètres sur lesquels on peut plus ou moins compter, une histoire et une mémoire, une volonté de transmettre. Elles laissent entrevoir que les opérateurs opèrent moins sur une machine qu'ils ne construisent avec elle une relation, à la manière de celle qu'on établit sur le long terme avec un «organisme complexe», vieillissant, ayant ses sautes d'humeur et requérant des efforts constants pour être «compris» (KnorrCetina 1999: 116-120). Certaines opérations, comme celles qui consistent à «remplir» et «mettre en collision», ont beau être «très standardisées", elles ne sont jamais parfaitement solubles dans une procédure. La machine 
bouge effectivement, parce que la terre bouge et que le sol est meuble; les portes s'ouvrent les jours de tempête et débraient l'ensemble du système; des hommes de chantier en surface piochent et interrompent la connexion. Et d'expérience, les protons sont sensibles à la manière dont ils sont injectés. Pas besoin de sauter dans le vide pour joindre les deux bouts des particules et du cosmos: il y a une vie entre les deux, sensible là.

\section{Le micron et le terramètre}

Janvier 2012. Service de métrologie directionnelle. Les géomètres qui y travaillent, collègues des géomètres de surface dont nous avons aperçu les activités précédemment, ont à charge la métrologie et l'alignement des aimants du LHC. L'opération d'alignement est capitale en ce qu'elle assure l'obtention de «beaux faisceaux », qui sont ceux susceptibles de produire des données fiables sur la structure du cosmos. «Ça se fait suivant des techniques un peu standard ", m'explique Michel, qui dirige le service. Appelés à opérer dans le tunnel, cent mètres sous terre, ces géomètresci interviennent lorsque le LHC est en arrêt - lorsque la machine est en marche, elle génère de la radioactivité impropre à toute présence humaine. C'est donc dans le creux du LHC, en quelque sorte, lorsqu'il est mis en veille, que les équipes du service de métrologie travaillent physiquement à l'alignement. L'autre partie de leur temps est consacrée au monitoring de l'installation qui, par son emprise territoriale, bouge dans le sol et subit également l'influence de la Terre, du mouvement des planètes et des étoiles - mouvements qu'il faut encore modéliser précisément de manière à toujours savoir «où se trouve la machine».

«Les détecteurs, commence Michel, sont constitués, entre autres, de trois aimants de focalisation qu'on appelle des quadripôles. On a installé, sur ces quadripôles, un système de monitoring permanent. Ce qui nous intéresse, c'est de connaitre la position des aimants les uns par rapport aux autres et leur position par rapport au détecteur. » Au moment de la construction du tunnel destiné à accueillir le LHC, un réseau de points géodésiques était utilisé pour aligner les aimants sur un rayon de cent kilomètres autour du CERN. «Ces points ont été mesurés dans les années 1970 avec les instruments les plus précis qu'on avait à l'époque, comme le terramètre, qui n'existait qu'en un ou deux exemplaires dans le monde. "Au milieu des années 1980 sont apparues les techniques GPS. Mais l'affaire se complique car les coordonnées dont on veut s'assurer doivent être prises cent mètres sous terre: «On a descendu des fils à plomb par les huit points sur le ring, avec des lunettes nadiro-zénithales. Avec cette technique, on arrive à descendre un point en surface cent mètres plus bas avec une marge d'un ou deux millimètres. À cette époque, on faisait aussi des mesures giro-théodolites, en utilisant un appareil qui mesure les angles en déterminant trois points. Mais ça demande une précision infinie parce que, si on a une erreur infime, elle se répercute de proche en proche et après on finit par se retrouver avec plusieurs centimètres d'écart... Donc on doit mesurer les angles par rapport au nord géographique, comme ça l'erreur n'est pas répercutée parce que le nord n'a pas bougé. " On commence ici à s'éloigner considérablement de la plaine de Gex, de ses forêts, de ses sangliers... Anticiper la course de particules au dixième de millimètre près requiert de situer l'installation au regard d'un point fixe qui ne peut être que très lointain. 


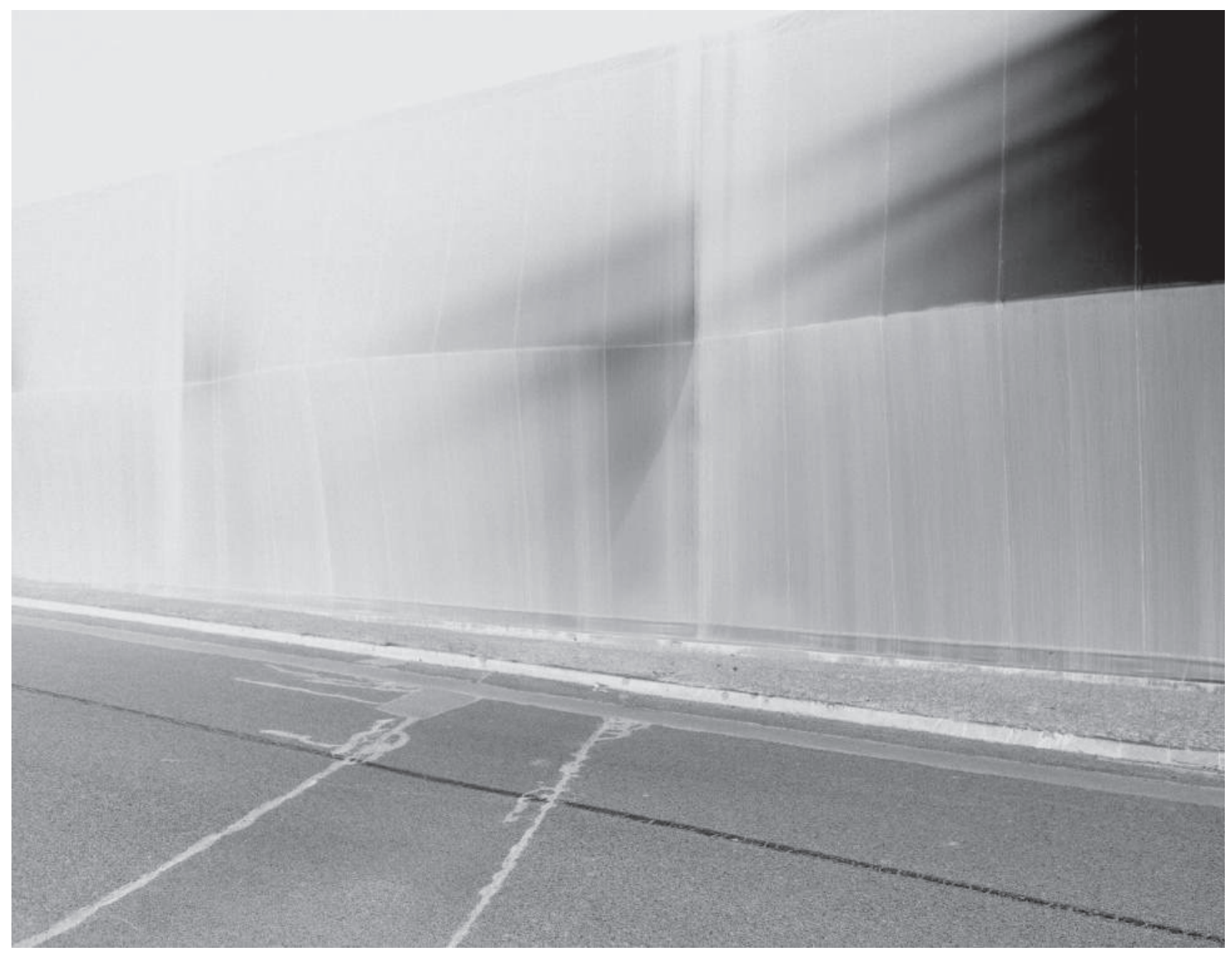

\section{ci dessus}

et ci-contre

fig. 8 et 9

Propagation de la

Monotonie, CERN, 2011

(C) Grégoire Eloy / F93. 


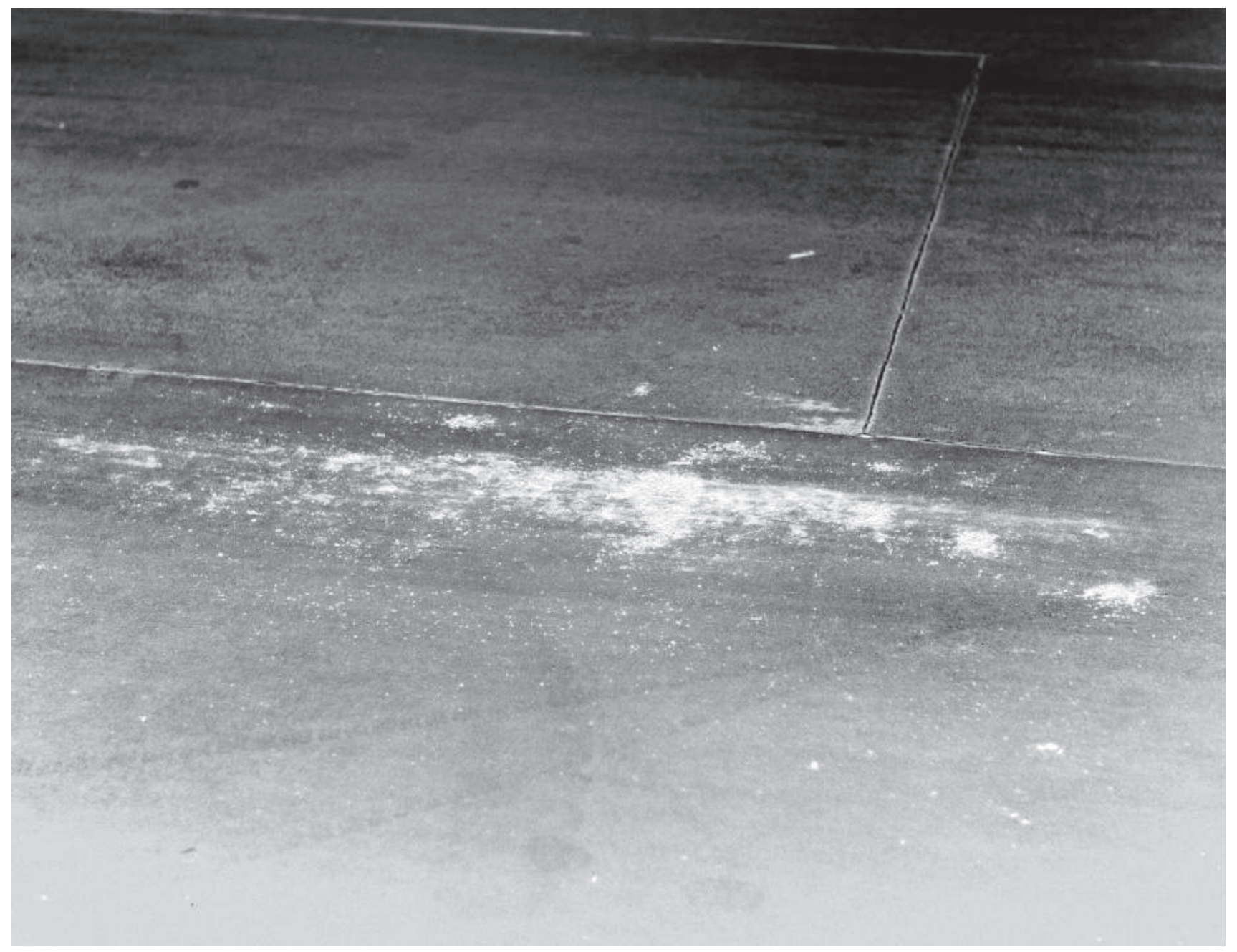


«Les physiciens dessinent une machine dans un plan local. Mais le problème auquel on se trouve confronté, souligne Michel, c'est qu'on est sur un plan de $10 \mathrm{~km}$ par $10 \mathrm{~km}$. " Le premier collisionneur de particules installé au CERN dans les années 1950, le PS, «était dans un plan. Point. Pour le SPS, le collisionneur suivant, on a commencé à observer qu'il y avait des distorsions dont on devait tenir compte. " Pour le LEP, le collisionneur pour lequel le tunnel circulaire long de $27 \mathrm{~km}$ a été construit et qui abrite aujourd'hui le LHC, «on mesurait les huit cents aimants quadripolaires tous les ans. Mais pour vous donner une idée, après la construction du LEP dans les années 1980, ils ont attendu plusieurs années avant de refaire une mesure et se sont rendu compte qu'ils avaient $18 \mathrm{~mm}$ de trou, ça commençait à perturber la physique, ce sont d'ailleurs les physiciens qui sont venus nous trouver et nous ont demandé de vérifier. Tous les ans, il y a un affaissement d'un millimètre et demi. »

L'équipe de Michel a donc la tâche considérable de donner aux physiciens les paramètres de transformation qu'ils vont utiliser en plan cartésien.

Les physiciens ne veulent pas subir les transformations de la Terre. II faut tenir compte des déviations de la verticale, et on fait ça en étudiant la modélisation de la forme de la Terre. On doit pouvoir déterminer le géoïde à 30 ou 40 microns près, et ça n'a jamais été fait avec une telle précision... On fait des tests là, à Villeneuse: on a loué des terrains aux paysans et on a installé, dans un tunnel où il n'y a pas d'éléments dedans, un point tous les $10 \mathrm{~m}$, on fait des mesures par rapport aux étoiles. On a loué pendant deux ans à un agriculteur du coin. On a aussi chez nous une personne qui s'est intéressée à l'influence des marées. On a des points dénivelés entre un côté du LHC et un autre, et on a installé, au-dessus des aimants, un tuyau, un réseau d'eau déminéralisée, qui relie tout sur $120 \mathrm{~m}$ de part et d'autre des expériences du LHC. Et on a en plus un réseau d'eau qui fait le tour de la caverne. Ces réseaux sont contrôlés en permanence et on peut déterminer grâce à eux un certain nombre de positions. On arrive ainsi à savoir où est l'expérience. Le problème, c'est que l'eau est perturbée par les marées: sur nos monitorings, on voit toutes les douze heures la forme des marées! Donc on doit mesurer l'influence de l'eau dans le tuyau et aussi sur les aimants. La Terre bouge, les aimants aussi. Donc il faut connaître les mouvements des aimants eux-mêmes et bouger les aimants pour qu'ils restent dans l'alignement.

Pour aligner les pièces de la machine entre elles, poursuit Michel, l'idéal serait de pouvoir tendre un fil sur $20 \mathrm{~km}$, mais ça, on ne sait pas faire. Le mieux qu'on ait fait, c'est de tendre un fil sur $500 \mathrm{~m}$, mais c'est déjà très difficile, il faut des fils spéciaux, fragiles et qui cassent facilement. Donc on part sur des fils de 200 m, en faisant des recoupements.

\section{ci-contre}

fig. 10

Propagation de la

Monotonie, CERN, 2011

(C) Grégoire Eloy / F93

\section{La bulle, la truite et le cosmos}

Janvier, toujours. J'ai rendez-vous à 8 h 30 au service de métrologie directionnelle pour descendre dans le tunnel avec Céline et Éric - c'est un grand jour pour moi, j'accède enfin aux «entrailles du cosmos», suivant 


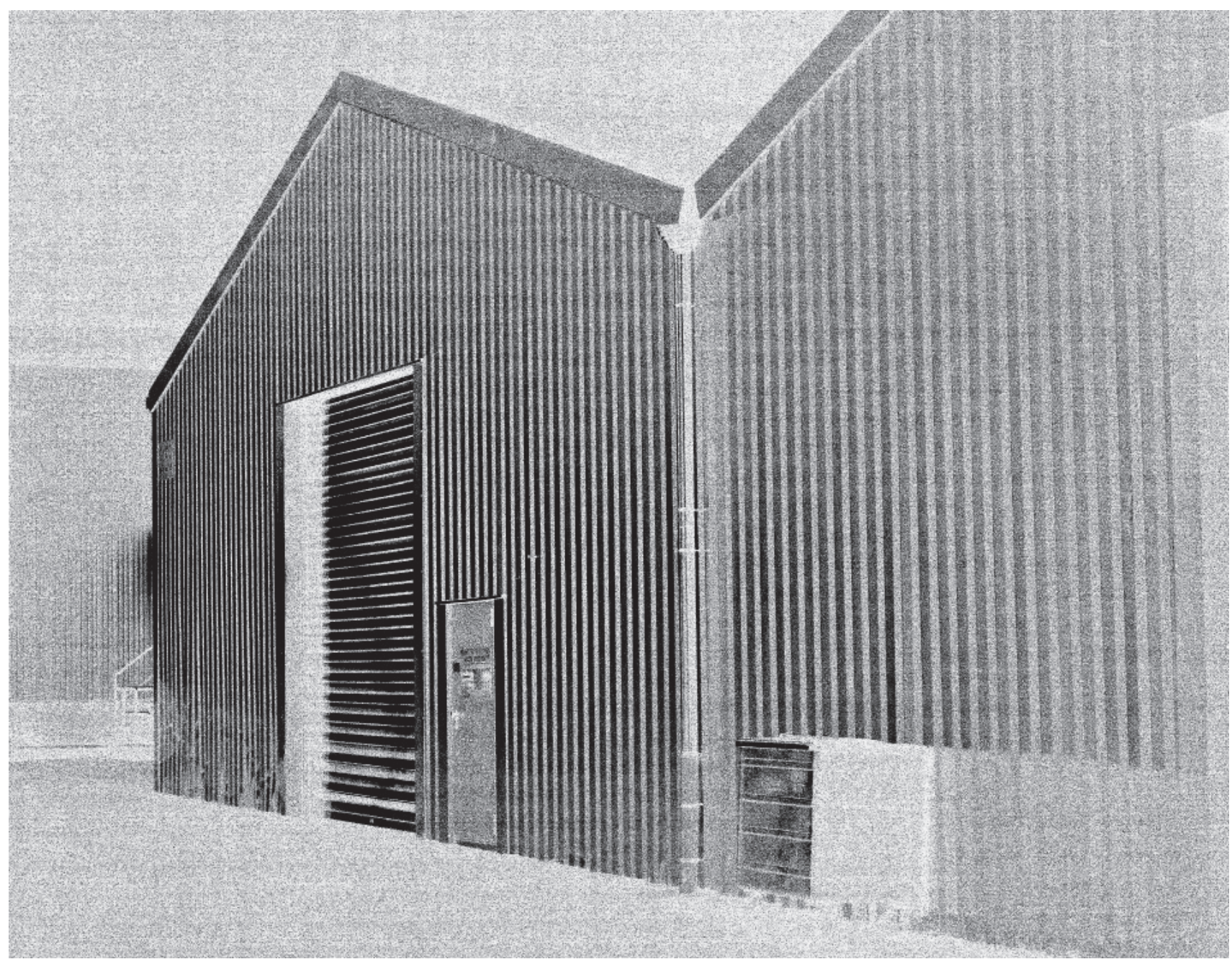


l'expression que j'entendrai plusieurs fois. Avant de partir, nous préparons le matériel: l'écartomètre d'un mètre, dans sa boîte en bois; ne pas oublier de prendre des piles; les casques et les «Biocell», les ceintures auxquelles sont accrochés les masques dont nous pourrions avoir besoin en cas de fuite d'hélium; les niveaux dont il faut encore «régler la bulle». Au rez-de-chaussée, dans un des ateliers du service, Céline calibre les niveaux sur la paillasse de marbre épais qui sert de plan de référence. Pour que la bulle soit centrée au mieux, elle la règle avec un pas de vis. Avec le temps, les bulles se dérèglent à cause des chocs durant les trajets ou bien des changements de température. «ll y a des limites mécaniques, physiques, à la précision des mesures! » s'exclame Céline.

Une fois descendus et parvenus au point T18 du tunnel, Céline et Éric commencent à installer le matériel. Ils ont pris deux diables avec lesquels ils déplacent deux colonnes transférées la veille dans le tunnel. L'une et l'autre servent de «base». Éric se charge de faire rouler l'une des colonnes cent mètres plus loin en remontant dans le tunnel tandis que Céline commence à fixer un fil sur l'autre. Dans sa main, une bonbonne de fil en nylon est déjà en place sur un support qui comprend aussi un moulinet. Une fois l'extrémité du fil fixée, Céline le déroule sur cent mètres à l'aide du moulinet. Ma surprise est totale: il s'agit de fil de pêche, de «pêche au gros". Et c'est bien un moulinet, acheté dans le commerce, comme en utilisent les pêcheurs - «pas les pêcheurs à la truite du dimanche, non plus! Mais bon, ce sont bien un fil à pêche et un moulinet! " Je suis déconcertée par la simplicité du dispositif utilisé pour mener à bien cette opération majeure qui consiste à aligner les aimants en résorbant les transformations mineures (1,5 $\mathrm{mm}$ par an) et les influences majeures (la rotondité de la Terre, l'influence des marées...) qui pourraient venir jouer sur la physique. Céline a entendu dire qu'il y avait des pêcheurs dans l'équipe dans les années 1960. Quelle ingéniosité! Compte tenu de la configuration de l'accélérateur, «il a bien fallu inventer des moyens... »

Une fois les colonnes installées et le fil tendu entre elles, Céline et Éric mettent en place l'écartomètre, fixé à l'une de ses extrémités sur un point fiduciel sur l'aimant. En faisant bouger sur la règle un petit écran surmonté d'un viseur optique, Éric vient placer la partie mobile de manière à ce que le fil soit sous le viseur. Pour se pencher sur le viseur, il doit monter sur une petite caisse en bois. Après quelques ajustements à l'œil, il me propose de regarder: au centre du viseur, il y a un cercle. Dessous, le fil dessine comme un trait (c'est d'ailleurs comme ça qu'ils l'appelleront de temps à autre). Viser consiste, pour Éric, à déplacer le viseur de manière à ce que le cercle repose sur le trait - à «trouver la tangente». L'écartomètre est utilisé pour se décaler par rapport aux points d'alésage, à l'extérieur de la courbure dessinée par les aimants. Céline dispose une bulle après l'autre sur le premier point d'alésage, qui va servir de référence. Au besoin, elle réajustera d'un très discret coup de vis le plan du point d'alésage. «Ça, c'est le juge de paix! » plaisante-t-elle au moment d'installer la plus grosse des deux bulles. «Bon... là c'est pas mal... En même temps, c'est une bulle très sensible et là on n'est pas au micron, on ne va pas pinailler. Elle est vraiment sensible, celle-là, je la connais bien, je peux la faire aller où je veux en la touchant un peu. » Éric, cent mètres plus loin, est en train de faire des réglages sur 
la colonne elle-même. Il tourne dans un sens, puis dans l'autre, les différentes manettes qui, à plusieurs endroits de la colonne, permettent de la stabiliser. II regarde la petite bulle témoin dont est munie la colonne. Équilibrer et stabiliser la colonne, en centrer la bulle, est une opération tout aussi délicate que de trouver la tangente dans le viseur.

À chaque station, il faut aussi régler la hauteur du fil, directement à partir de la colonne, afin qu'il vienne se glisser sous le viseur. À la fin d'une station, au moment d'enregistrer les données sur le petit moniteur, Éric et Céline consultent l'ensemble des mesures prises, ainsi que les écarts calculés par la machine sur la base de paramètres entrés préalablement. «Là, on a un écart de 1,8, c'est un peu beaucoup... La tolérance sur les dipôles du LHC, c'est de 0,2. C'est le déplacement toléré par rapport à la mesure théorique, faite au moment de la mise en place des aimants. Bon, de toute façon, c'est pour ça qu'on fait les mesures. Après ils vont passer le rabot. Allez, on fait la fermeture. » «L'année dernière, raconte Céline, un vendredi soir, on a laissé le matériel dans la voiture, on était fatigués. Le lundi matin, bon, il faisait froid, on a bien vu que les instruments étaient froids mais bon... On a fait des mesures pendant trois heures, sur une opération qui nous en prend habituellement quatre. On était presque au bout et on s'est rendu compte que nos mesures étaient fausses. De deux dixièmes! C'est vrai qu'à ce degré de précision, l'atmosphère, ça compte! »

Nous finissons une nouvelle station puis nous arrêtons pour déjeuner. On réinstalle ensuite le dispositif pour le troisième segment, en continuant à remonter le tunnel d'injection. Encore plus qu'à la station précédente, le fil au milieu passe largement au-dessus du viseur. II faut le détendre de beaucoup. Avec les courants d'air, si subtils soient-ils, il remonte, bouge, vacille. Impossible de le capturer dans le viseur ni de le stabiliser.

- Il faut que tu le redescendes un peu, que tu donnes encore du mou, et je me dépêche de faire la mesure. [Et, s'adressant au fil.] Oh là là, calme-toi, calmos... Ah non, mais là, je ne peux pas mesurer.... [Plus fort à l'adresse de Céline.] Pas possible, Céline, ça bouge trop!

- Comment ils ont fait, l'année dernière?

- Ils ont peut-être mis un poids sur le fil...

- Je vais demander à Patrick. [Au téléphone avec Patrick.] Tu sais, on est juste à l'endroit où il y a la pente, avant que ça ne remonte. Et le problème, c'est que le fil est tout droit, donc quand on arrive au centre... Si si, les colonnes sont déjà sécurisées... Non, c'est déjà tout en bas...

Éric et moi continuons de regarder le fil remonter doucement... «Ça a pris 2 ou $3 \mathrm{~cm}$, c'est pour ça que dans le LHC ils ont mis des gaines. » Céline poursuit au téléphone: «Éric proposait de mettre un poids... O.K., je retends et on met quelque chose sur le fil. " Après avoir cherché un moment dans son sac, Céline finit par poser son trousseau de clés sur le fil. Ils parviennent à régler la hauteur en déplaçant le poids le long du fil.

- Ah... ça bouge...

- Il faut laisser le fil se stabiliser. double page suivante

fig $=11$

Propagation de la

Monotonie, CERN, 2011

(c) Grégoire Eloy / F93. 


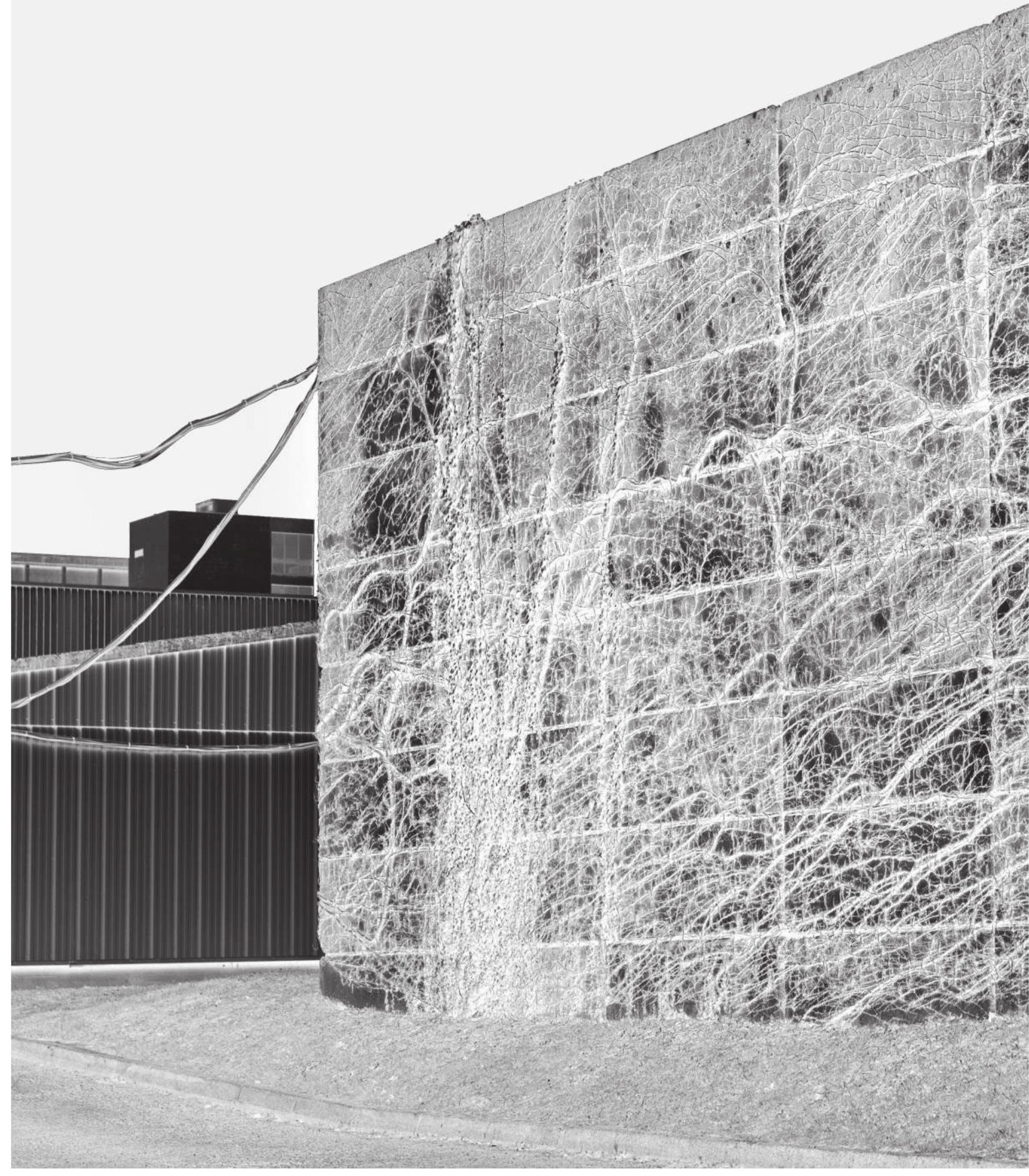




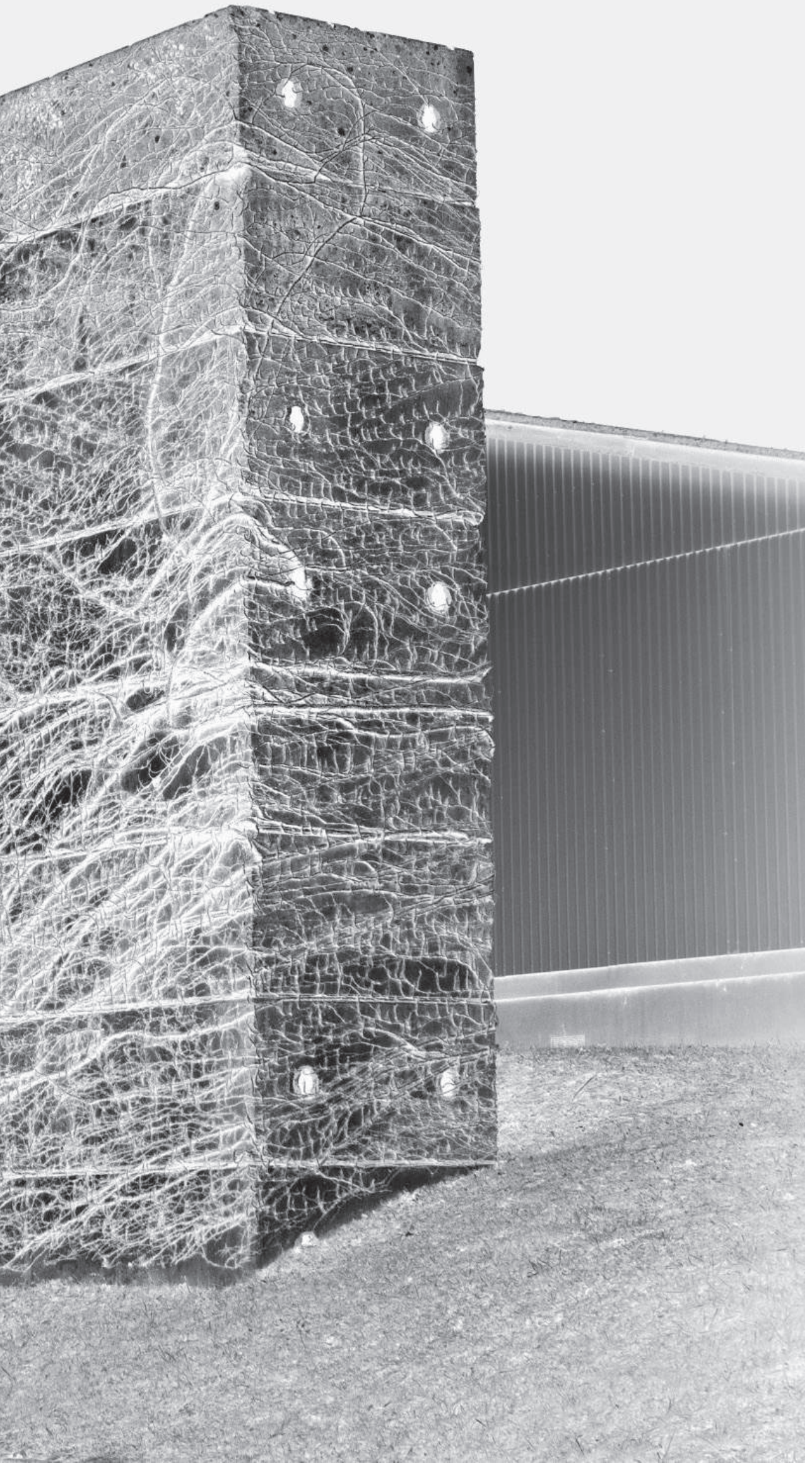


5. Entretien avec Fabiola Gianotti, http://cds.cern. $\mathrm{ch} / \mathrm{record} / 1223621 ? \mathrm{In}=\mathrm{fr}$ consulté le 13 juin 2011.
- On peut peut-être tendre un peu? et on bougera les clés.

- On n'a qu'à faire plusieurs lectures et on fera la moyenne.

Du bout du doigt, Céline stabilise les clés et Éric parvient enfin à prendre une mesure. Puis il décale l'écartomètre au point suivant. «ll faut que tu approches les clés. Recule un peu... top! » Bon an mal an, ils viennent à bout de cette nouvelle station. Nous avons passé du temps dans ce creux, et personne ne se sent le courage d'attaquer une nouvelle station. Ils démontent le fil, déplacent les colonnes qu'ils rangent dans une petite galerie connexe. Nous regagnons la surface.

\section{Conclusion}

Lorsqu'on décide de s'intéresser au LHC, à la machine non seulement dans ce qu'elle a nécessité comme travail ou dans les résultats qu'elle produit, mais en tant qu'elle-même, dans la matérialité qui la caractérise, on en arrive vite à se dire qu'elle est un territoire avant d'être un laboratoire. Travailler à sa maintenance, contrôler ce qu'elle émet, c'est parcourir un territoire et connaître ce qui le peuple. Lorsque Fabiola Gianotti, porte-parole de l'expérience Atlas, parle du LHC comme d'un «instrument d'exploration d'un territoire inconnu ${ }^{5}$ ", C'est à l'univers qu'elle se réfère, non au pays de Gex. Pourtant, impossible d'explorer l'un sans l'autre; impossible de se connecter au cosmos sans en passer par la vallée, les plaines sur lesquelles paissent les vaches, les cours d'eau, les couches sédimenteuses. Et impossible, même, d'établir entre les uns et les autres une quelconque hiérarchie. Toujours, le haut, le bas et le milieu, le cosmos, la surface et le sous-sol, le majeur et mineur doivent être pris en compte. Le physicien et historien des sciences Peter Galison conclut son ouvrage L'Empire du temps. Les horloges d'Einstein et les cartes de Poincaré de la manière suivante: "Les alchimistes-astrologues du Moyen Âge disaient: baissons les yeux pour voir le ciel, levons les yeux pour voir la terre. Cette conception du savoir nous convient à merveille. Car, en baissant les yeux (vers le réseau des horloges régulées de façon électromagnétique), nous voyons ce qui se passe en haut: la configuration des empires, la métaphysique et la société civile. En levant les yeux (vers la philosophie d'Einstein, vers la simultanéité de Poincaré), nous voyons ce qui se passe en bas: les fils conducteurs, les dispositifs instrumentaux et les impulsions électriques. " (Galison 2005 [2003]: 415) Galison montre que le concept de synchronie par lequel les deux physiciens, dans la première moitié du xx $x^{e}$ siècle, avaient à cœur de «mettre de l'ordre dans la mesure du temps et de l'espace» (ibid.: 17) a été élaboré au cours d'un processus technologique sophistiqué qui devait prendre en compte les propriétés de l'électricité, l'étendue du réseau ou encore le contrôle d'horloges éloignées dans l'espace - autant de composantes rendues complexes par le simple fait que la Terre tourne.

Je le comprends à suivre les opérateurs, les géomètres au travail: le LHC n'est pas une machine déconnectée de notre univers. Pour incommensurable que cela paraisse, ce dont elle traite - de la naissance et de la composition du cosmos, ou du zeptospace - est toujours relié de mille manières au lieu qui l'accueille et qu'elle investit. C'est une «machine romantique», suivant les termes de John Tresch, qui se reconnaît par les «fusions et échanges de propriétés [qui s'instaurent] entre les humains et leurs instruments" 


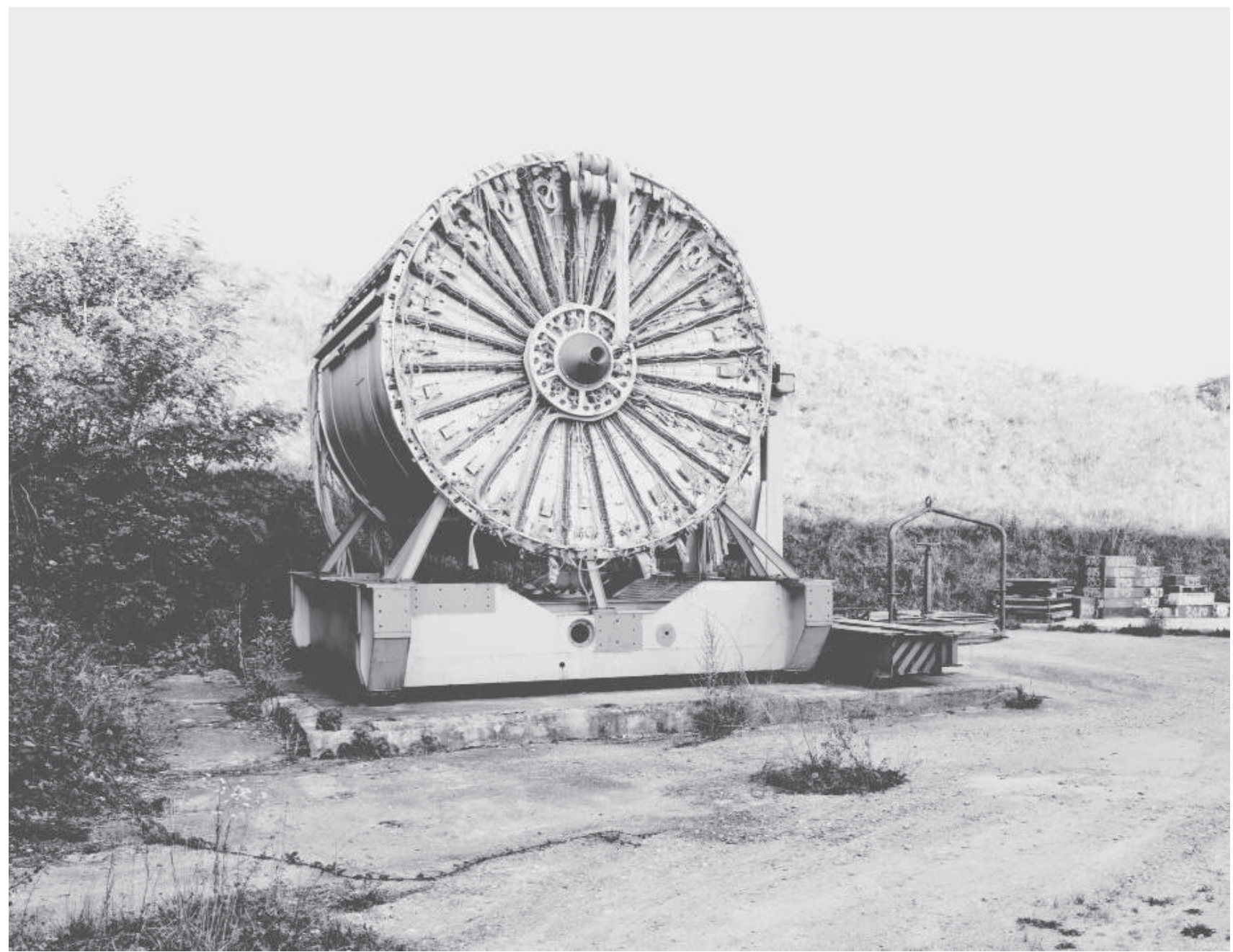

fig. 12

Propagation de la

Monotonie, CERN, 2011

(c) Grégoire Eloy / F93. 


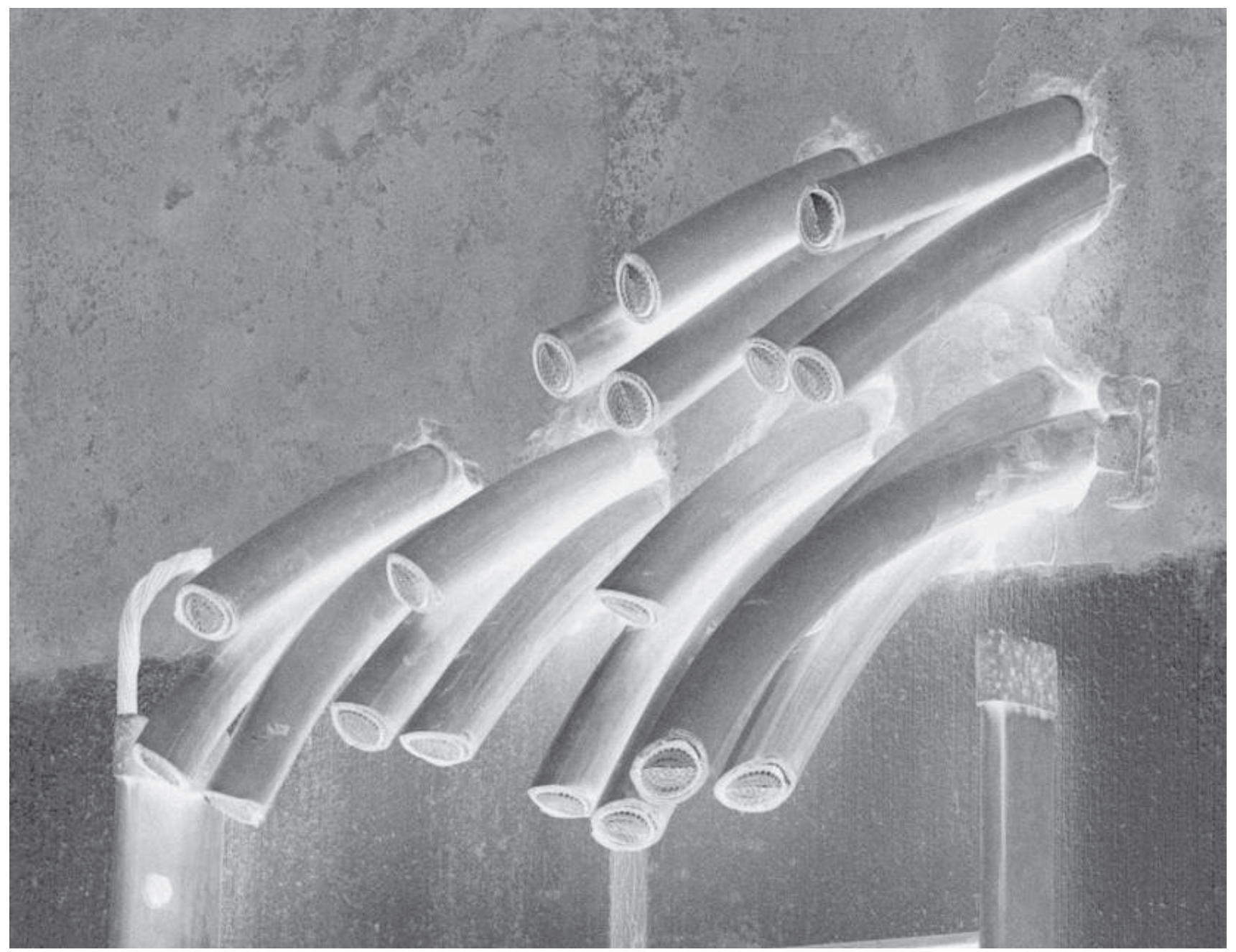

fig. 13

Propagation de la

Monotonie, CERN, 2011

(C) Grégoire Eloy / F93. 
(Tresch 2012: 12, notre traduction). La profusion des instruments de captation et de mesure, des dispositifs de précision, n'embarque pas vers un surplus d'objectivité qui nous éloignerait d'autant de la sensibilité et du savoir-faire pratique des physiciens de la machine et des opérateurs (Daston et Galison 2012). Le LHC constitue une «technologie charismatique» dans laquelle se manifestent tant une «routinisation de l'enchantement» qu'un «enchantement de la routine» (Tresch 2012: 14, notre traduction): c'est cela seulement qui peut rendre compte de la fascination extatique qu'il exerce sur ceux qui s'en approchent, sur ceux, plus particulièrement, qui ont été témoins de sa venue au monde. Comme pour ces «machines romantiques» qui intéressent Tresch et qui peuplent le XIX siècle des fantasmagories qu'elles drainent, les gens que j'ai rencontrés reconnaissent volontiers au LHC, «non pas tant [la capacité] de détruire l'aura ou de précipiter le désenchantement du monde, [...] mais plutôt le pouvoir étrange d'animer l'inanimé, d'émanciper et de spiritualiser la matière vibrante» (ibid. : 16, notre traduction).

C'est davantage par la machine, le dispositif technique lui-même (son échelle et la gestion de celle-ci), que par la physique et les particules que j'acquiers le sentiment profond que le LHC me concerne et a quelque chose à me dire de la place que j'occupe dans l'univers: à son contact, j'ai eu bel et bien le sentiment d'être dans l'univers, de me situer à l'aune de cette échelle-ci. Le pays de Gex est un des points d'accès au cosmos, par le LHC. C'est le LHC qui me donne à voir et à sentir que je m'inscris dans un réseau de coordonnées au sein de l'univers lui-même. En le saisissant ainsi, en me situant au niveau du sol quelque part entre cosmos et particules, je vois, à la place de l'éloquence - qui vient et sied naturellement au dispositif expérimental le plus grand du monde -, de menues actions s'accomplir; j'observe des tressaillements machiniques et des vibrations infinitésimales; j'entends parler de précisions défaillantes, de respirations subtiles. Et tout cela contribue à exciter, plutôt qu'à réfréner, mon appétit cosmologique. Pour être capable de se penser dans l'univers, il faut toujours savoir où en sont les blaireaux et les sangliers qui courent les chemins forestiers le long desquels circulent aussi les réseaux électriques et de fibres qui alimentent la machine; il faut avoir un regard aussi sur les activités des hommes et leurs préoccupations. Partant, en rendant visible la place qu'ils occupent dans le dispositif, il s'agit de faire valoir l'idée que se connecter au cosmos ne vaut que si l'on parvient à le faire avec tous ceux qui habitent notre magma anthropologique depuis longtemps: les animaux, les hommes et leurs affaires mondaines, les dieux même.

CNRS, Laboratoire d'ethnologie et de sociologie comparative sophie.houdart@mae.u-paris10.fr figures pages $24,28,30-31$,

$38,39,41,44-45,47$ et 48

Les photographies en noir et blanc reproduites dans cet article ont été réalisées lors d'une résidence de Grégoire Eloy au CERN en 2011, dans le cadre du projet collectif «Propagation de la Monotonie" produit par F93, Centre de culture scientifique et technique de Seine-Saint-Denis. www.gregoireeloy.com 
Bibliographie

\section{Beech, Martin}

2010 The Large Hadron Collider.

Unraveling the Mysteries of the

Universe. New York, Springer.

\section{Daston, Loraine} et Galison, Peter

2012 Objectivité. Paris, Les Presses du Réel.

\section{Galison, Peter}

2005 [2003] L'Empire du temps. Les horloges d'Einstein et les cartes de Poincaré. Paris, Gallimard.

\section{Giudice, Gian Francesco}

2010 A Zeptospace Odyssey. A Journey into the Physics of the $L H C$. New York, Oxford University Press.

\section{Helmreich, Stefan}

2009 "Intimate sensing", in Sherry Turkle (éd.), Simulation and Its Discontents. Cambridge, The MIT Press : 129-150.

\section{Houdart, Sophie}

2015 Les Incommensurables.

Bruxelles, Zones Sensibles.

\section{Hutchins, Edwin}

1995 Cognition in the Wild.

Cambridge et Londres,

The MIT Press.

\section{Ingold, Tim}

2000 The Perception

of the Environment. Essays in

Livelihood, Dwelling and Skill.

Londres et New York, Routledge.

2011 Being Alive. Essays

on Movement, Knowledge

and Description. Londres

et New York, Routledge.

\section{Knorr-Cetina, Karin}

1999 Epistemic Cultures. How the Sciences Make Knowledge. Cambridge et Londres, Harvard University Press.
Petits récits destinés à joindre les deux bouts des particules au cosmos - en passant par la Suisse. Par Sophie Houdart

\section{Koestler, Arthur}

1945 Le Zéro et l'Infini. Paris,

Calmann-Lévy.

\section{Latour, Bruno}

2014 «L'Anthropocène et la destruction de l'image du globe ", in Émilie Hache (éd.) De l'univers clos au monde infini. Paris, Éditions Dehors : 29-56.

\section{Stengers, Isabelle}

1996 La Guerre des sciences. Cosmopolitique I. Paris,

La Découverte-Les Empêcheurs de penser en rond.

\section{Traweek, Sharon}

1988 Beamtimes and Lifetimes. The World of High Energy

Physicists. Cambridge et Londres, Harvard University Press.

\section{Tresch, John}

2012 The Romantic Machine. Utopian Science and Technology After Napoleon. Chicago et Londres, The University of Chicago Press.

\section{Vermorel, Henri et Vermorel, Madeleine}

1993 Sigmund Freud et Romain Rolland. Correspondance 1923-1936. Paris, PUF. page 106 et ci-contre

Propagation de la Monotonie, CERN 2011 (c) Grégoire Eloy / F93.

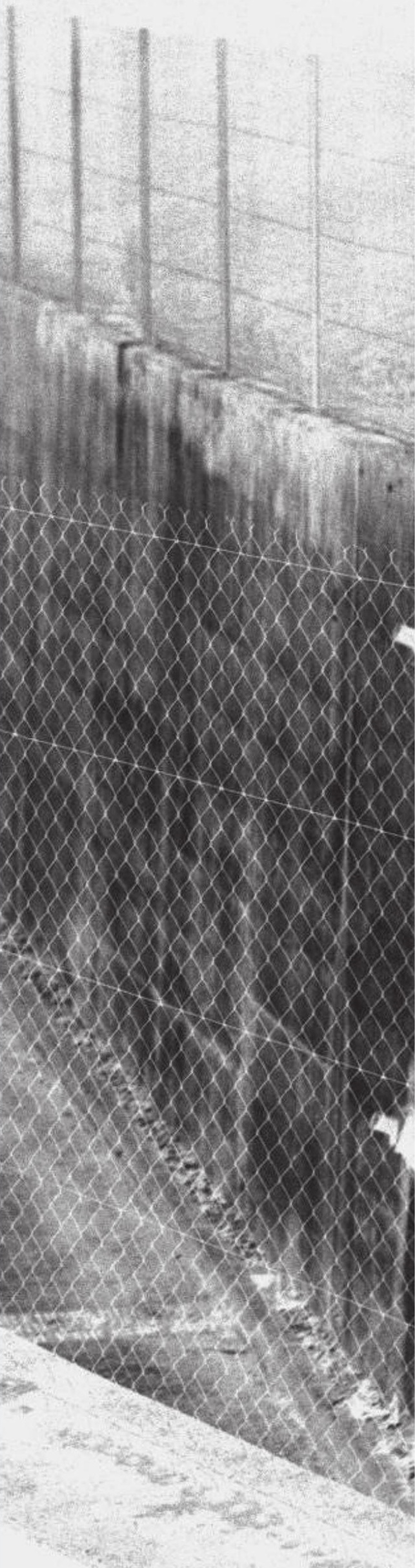


